\title{
On the astronomical origin of the Hallstatt oscillation found in radiocarbon and climate records throughout the Holocene
}

\author{
Nicola Scafetta ${ }^{1 \dagger}$, Franco Milani ${ }^{2}$, Antonio Bianchini ${ }^{3,4}$, Sergio Ortolani ${ }^{3,4}$
}

August 8, 2018

\author{
${ }^{1}$ Department of Earth, Environmental and Resources Science, Università degli Studi di Napoli Federico II, \\ Napoli, Italy \\ ${ }^{2}$ Astronomical Association Euganea, Padova, Italy \\ ${ }^{3}$ Department of Physics and Astronomy, University of Padova, Italy \\ ${ }^{4}$ INAF-Osservatorio Astronomico di Padova, Vicolo dell'Osservatorio 5, I-35122 Padova, Italy \\ †email: nicola.scafetta@unina.it
}

\begin{abstract}
An oscillation with a period of about 2100-2500 years, the Hallstatt cycle, is found in cosmogenic radioisotopes $\left({ }^{14} \mathrm{C}\right.$ and $\left.{ }^{10} \mathrm{Be}\right)$ and in paleoclimate records throughout the Holocene. This oscillation is typically associated with solar variations, but its primary physical origin remains uncertain. Herein we show strong evidences for an astronomical origin of this cycle. Namely, this oscillation is coherent to a repeating pattern in the periodic revolution of the planets around the Sun: the major stable resonance involving the four Jovian planets - Jupiter, Saturn, Uranus and Neptune - which has a period of about $p=2318$ yr. Inspired by the Milanković's theory of an astronomical origin of the glacial cycles, we test whether the Hallstatt cycle could derive from the rhythmic variation of the circularity of the solar system disk assuming that this dynamics could eventually modulate the solar wind and, consequently, the incoming cosmic ray flux and/or the interplanetary/cosmic dust concentration around the Earth-Moon system. The orbit of the planetary mass center (PMC) relative to the Sun is used as a proxy. We analyzed how the instantaneous eccentricity vector of this virtual orbit varies from 13,000 B. C. to 17,000 A. D.. We found that it undergoes kind of pulsations as it clearly presents rhythmic contraction and expansion patterns with a 2318 yr period together with a number of already known faster oscillations associated to the planetary orbital stable resonances. There exists a quasi $\pi / 2$ phase shift between the $2100-2500$ yr oscillation found in the ${ }^{14} C$ record and that of the calculated eccentricity function. Namely, at the Hallstatt-cycle time scale, a larger production of radionucleotide particles occurs while the Sun-PMC orbit evolves from more elliptical shapes $(e \approx 0.598)$ to more circular ones $(e \approx 0.590)$, that is while the orbital system is slowly imploding or bursting inward; a smaller production of radionucleotide particles occurs while the Sun-PMC orbit evolves from more circular shapes $(e \approx 0.590)$ to a more elliptical ones $(e \approx 0.598)$, that is while the orbital system is slowly exploding or bursting outward. Since at this timescale the PMC eccentricity variation is relatively small $(e=0.594 \pm 0.004)$, the physical origin of the astronomical $2318 \mathrm{yr}$ cycle is better identified and distinguished from faster orbital oscillations by the times it takes the PMC to make pericycles and apocycles around the Sun and the times it takes to move
\end{abstract}


from minimum to maximum distance from the Sun within those arcs. These particular proxies reveal a macroscopic 2318 yr period oscillation, together with other three stable orbital resonances among the outer planets with periods of 159, 171 and $185 \mathrm{yr}$. This $2318 \mathrm{yr}$ oscillation is found to be spectrally coherent with the $\Delta^{14} C$ Holocene record with a statistical confidence above $95 \%$, as determined by spectral analysis and cross wavelet and wavelet coherence analysis. At the Hallstatt time scale, maxima of the radionucleotide production occurred when, within each pericycle-apocycle orbital arc, the time required by the PMC to move from the minimum to the maximum distance from the Sun varies from about 8 to 16 years while the time required by the same to move from the maximum to the minimum distance from the Sun varies from about 7 to 14 years, and vice versa. Thus, we found that a fast expansion of the Sun-PMC orbit followed by a slow contraction appears to prevent cosmic rays to enter within the system inner region while a slow expansion followed by a fast contraction favors it. Similarly, the same dynamics could modulate the amount of interplanetary/cosmic dust falling on Earth. Indeed, many other stable orbital resonance frequencies (e.g. at periods of $20 \mathrm{yr}, 45 \mathrm{yr}, 60 \mathrm{yr}, 85 \mathrm{yr}, 159-171-185 \mathrm{yr}$, etc.) are found in radionucleotide, solar, aurora and climate records, as determined in the scientific literature. Thus, the result supports a planetary theory of solar and/or climate variation that has recently received a renewed attention. In our particular case, the rhythmic contraction and expansion of the solar system driven by a major resonance involving the movements of the four Jovian planets appear to work as a gravitational/electromagnetic pump that increases and decreases the cosmic ray and dust densities inside the inner region of the solar system, which then modulate both the radionucleotide production and climate change by means of a cloud/albedo modulation.

Keywords: Analysis of ${ }^{14} C$ radionucleotide records; Origin of the 2100-2500 years Hallstatt oscillation; The 2318-year Jupiter-Saturn-Uranus-Neptune stable resonance; Holocene paleoclimatic records; Identification and modeling of solar and planetary oscillations; Spectral and wavelet coherence analysis.

Cite: Scafetta, N., Milani, F., Bianchini, A., Ortolani, S.: On the astronomical origin of the Hallstatt oscillation found in radiocarbon and climate records throughout the Holocene. Earth-Science Reviews 162, 24-43, 2016. http://dx.doi.org/10.1016/j.earscirev.2016.09.004

\section{Introduction}

Cosmic rays continuously collide with the Earth's atmospheric molecules fragmenting their nuclei and producing neutrons. The collisions of thermal neutrons with nitrogen nuclei ${ }_{7}^{14} \mathrm{~N}$ made of 7 protons and 7 neutrons) give origin to cosmogenic radioisotopes $\left({ }_{6}^{14} C\right.$ made of 6 protons and 8 neutrons) according to the following reaction: $n+{ }_{7}^{14} N \rightarrow{ }_{6}^{14} \mathrm{C}+p .{ }^{14} \mathrm{C}$ rapidly reacts with oxygen to produce $\mathrm{CO}_{2}$ and, as such, is absorbed by biological organisms such as trees and marine corals. Since their formation and their capture by biological systems, ${ }^{14} \mathrm{C}$ atoms undergo a radioactive beta decay into stable ${ }^{14} \mathrm{~N}$ atoms with a half-life time of 5730 years according to the following reaction: ${ }_{6}^{14} C \rightarrow{ }_{7}^{14} N+e^{-}+\bar{\nu}_{e}$. Thus, by measuring the percent of ${ }^{14} C$ atoms present in a specific organic material, if the age of the latter can be timed independently, it is possible to determine the ${ }^{14} \mathrm{C}$ atmospheric concentration of the past. Determining this time series is astronomically and geophysically important because ${ }^{14} C$ concentration variations are a direct consequence of changes in the intensity of the cosmic ray flux reaching the Earth, in solar magnetic activity (Bard et al., 1997; Stuiver and Quay, 1980), in the Earth's dipole moment (Elsasser et al., 1956, Lal, 1988, O’Brien, 1979) and in a number of parameters regulating the radiocarbon exchange system (Oeschger et al., 1975: Siegenthaler et al., 1980. Stocker and Wright, 1996, Goslar et al., 1999).

Several experimental evidences demonstrate that ${ }^{14} \mathrm{C}$ concentration varies in time (e.g. Damon and Linick, 1986; Kromer et al., 1998, and many others). Bray (1968), using short records, and later Houtermans (1971) using records spanning throughout the Holocene (since 10,000 B. C.), noted that ${ }^{14} C$ concentration 
has changed cyclically with the longest certain period being about 2100-2500 years long. Longer oscillations could be present, but the record was too short to detect them. This period is known in the literature as the Hallstatt cycle (Vasiliev and Dergachev, 2002) because its cooling minimum occurred before the Maunder Minimum [1645:1715] happened about 2800 years ago during a late Bronze - early Iron cultural transition in an Austrian archaeological site located at Hallstatt. Other major oscillations found in the ${ }^{14} \mathrm{C}$ concentration records have periods of about 900-1050 year (Bond et al. 2001, Kerr, 2001, Scafetta, 2012a; Davis and Bohling, 2001) known in the literature as the Eddy cycle (cf. McCracken et al., 2013) and a 208-year cycle known in the literature as the Suess or de Vries cycle (Sonett, 1984). The presence of fundamental harmonics in radiocarbon records have been confirmed by numerous studies Abreu et al., 2012, Damon and Linick, 1986; Damon, 1988, Damon et al., 1990, Damon and Sonett, 1992, Damon and Jirikowić, 1992, McCracken et al. 2013, Vasiliev and Dergachev, 1998, 2002).

An oscillation with a period of about 2100-2500 years has been found also in a number of paleoclimate records and/or events throughout the 12,000 years of the Holocene (e.g.: Levina and Orlova, 1993; O'Brien et al. 1995). For example, it was found in the $\delta^{18} O$ concentration measured in ice cores and in deep-see cores with high sedimentation rates (Pestiaux et al. 1988). Dendroclimatological considerations have also demonstrated that the Little Ice Age (1500-1800 year A.D.), the Hallstattzeit cold epoch (750-400 year B.C.) and the earlier cold epoch (3200-2800 year B.C.) are separated by 2100-2500 years (Damon and Sonett, 1992 , p. 378). Given the evident correlation and synchronicity between the 2100-2500 year oscillation found in the ${ }^{14} \mathrm{C}$ concentration record and in a number of paleoclimatic data, all these records must be linked together.

Climate variations and ocean/air ventilation changes could also modulate the production of ${ }^{14} C$ concentration. However, this interpretation leaves unanswered the question of why the climate would oscillate with a 2100-2500 year cycle. ${ }^{14} \mathrm{C}$ concentration could also vary because of changes in the Earth's magnetic field shielding the Earth from incoming cosmic rays (e.g. Damon and Linick, 1986). However, changes of the Earth's dipole field may be too weak to cause the 2100-2500 year oscillation in the radiocarbon records (cf.: Creer, 1988: Damon and Sonett, 1992).

Also cosmic rays, which directly form cosmogenic radioisotopes, influence the Earth's climate. Indeed, numerous empirical evidences and theoretical considerations have pointed out that cosmic rays can contribute to the formation of clouds and, therefore, modulate the Earth's albedo by ionizing the atmosphere (e.g.: Kirby, 2007, Svensmark, 1998; Svensmark et al., 2009, 2012, Tinsley, 2008), although cosmic rays alone may not explain the full amount of atmospheric precipitation variation. The existence of an astronomical origin of the involved mechanisms would also be supported by the finding that variations in ${ }^{14} C$ concentration are correlated with the solar system's galactic motion and imprinted in the Phanerozoic climate over the last 600 million years (e.g.: Shaviv et al., 2014).

Several authors have concluded that the observed 2100-2500 year oscillation both in the cosmogenic radioisotope records and in the climate records has a solar origin (e.g.: Dergachev and Chistyakov, 1995. Hood and Jirikowić, 1990, Hoyt and Schatten, 1997). Indeed, ${ }^{14} C$ records, as well as ${ }^{10} B e$ records reproduce features present in the sunspot number records such as the Maunder and Dalton solar minima, and other solar directly observed patterns (cf.: Adolphi et al. 2014, Bard et al., 1997, 2000, Scafetta, 2012a, Steinhilber et al. 2009 Usoskin et al. 2016). However, these considerations still do not explain why solar activity should vary with a 2100-2500 year oscillation. Indeed, this oscillation might also be forced on the system.

In any case, even if cosmic rays are one of the drivers of climate change, one should explain why they are modulated by a 2100-2500 years periodicity. The origin of this can be of three kinds: astronomical, solar, or Earth's endogenous. Gregori (2002) suggested that the encounters of the Solar System with clouds of interstellar matter modulate solar physics, hence its activity, and also its release of solar wind. The Earth, with its magnetosphere, captures a fraction estimated at $\sim 0.5 \times 10^{-9}$ of the surface, at $1 \mathrm{AU}$, of the expanding solar corona. That is, this is a very tiny fraction of the whole volume of the out-flowing solar 
wind. Similarly, the solar system is presumably capturing a very tiny fraction of the clouds of interstellar matter. These records are expected to be erratic and/or, on multi-million year time scale, they can also be modulated by the movement of the solar system within the galaxy (cf.: Gregori, 2002, Shaviv et al., 2014). However, at the shorter time scales the incoming dust flux might be also modulated by the internal oscillating dynamics of the solar system. Hence, a long term solar modulation could be only indicative of a galactic modulation of solar physics.

Herein we hypothesize that the 2100-2500 yr oscillation in the radiocarbon records has an astronomical origin, and search whether an astronomical record clearly manifests such an oscillation. In this regard, Charvátová (2000) was the first in suggesting that the observed 2100-2500 yr oscillation could be caused by the solar inertial motion, that is by the wobbling of the Sun around the barycenter of the solar system due to the orbital movements of its planets. She proposed a simplified model where the 2100-2500 yr oscillation had to be on average 2402.2 years long, which would corresponds to the Jupiter/Heliocenter/barycenter alignments $(9.8855 \times 243=2402.2$ year $)$. About the secular solar oscillations Charvátová $(2000)$ showed that the inertial motion of the Sun varies from a trefoil ordered state, where the orbital patterns nearly repeat while rotating relative to the fixed stars, to a disordered one, where the orbits show confused and chaotic patterns. The ordered cases correspond to stable patterns correlated with historical solar maxima while the disordered ones correlate with historical solar minima such as the Wolf minimum (1280 to 1340), the Spörer minimum (1420 to 1570), the Maunder minimum (1645 to 1715) and the Dalton minimum (1790 to 1820). Based on these patterns, the current period (1985 to 2040) could yield to a Dalton like minimum (cf.: Mörner, 2015, Scafetta, 2010, 2012a). Moreover, again using a simplified model, Scafetta (2012c) showed that the conjunctions of Jupiter and Saturn, using their tropical orbital periods, fully precess over a quasi 2400 year period.

However, the above models were oversimplified as they neglected the presence of the other planets: they could be unconvincing because the 2100-2500 yr oscillation was merely implicit in calculations whose physical meaning was hypothetical. In general, it could be argued that it is physically unlikely that the solar inertial motion could be the direct cause of a variation in the solar activity because the Sun is in free-fall in it and should not feel it. A more realistic hypothesis requires that the solar inertial motion is mathematically linked to some physical mechanism yielding a variability in solar magnetic activity and/or in the incoming cosmic ray flux. The solar inertial motion could be just a proxy collecting the relevant information about the dynamics of the solar system. In principle, the planetary motion can produce gravitational and/or electromagnetic forcings directly onto the Sun, interacting with its magnetic activity, and/or within the heliosphere. In this way, it could be modulating the incoming flux of cosmic rays as well as the concentration of the interplanetary/cosmic dust around the Earth-Moon system (Ermakov et al., 2009a; Ollila, 2015). Such forcing should then maintain the imprinting of its origins and be synchronized with some planetary resonances.

Some physical mechanisms explaining a planetary modulation of solar and climate activity are currently investigated (Abreu et al., 2012, Scafetta, 2012b, Scafetta and Willson, 2013b, Wolff and Patrone, 2010). A planetary origin of solar and climate oscillations, which has been proposed since antiquity, does have numerous empirical evidences and some preliminary explanations (e.g.: Abreu et al., 2012, Charvátová, 2009: Cionco and Soon, 2014, Hung, 2007; Fairbridge, 1984, Fairbridge and Sanders, 1987; Jakubcová and Pick, 1986; Jose, 1965; McCracken et al., 2013, 2014, Mörner, 2013, 2015, Mortari, 2010, Puetz et al., 2014, Salvador, 2013; Scafetta, 2010, 2012a|b, 2013, 2014a, 2016, Scafetta and Willson, 2013ab, Sharp, 2013, Solheim, 2013, Tan and Cheng, 2013, Tattersall, 2013a b; Wilson, 2013).

The present work aims to provide a robust evidence that the 2100-2500 yr Hallstatt oscillation found both in cosmogenic radioisotopes and in climate records throughout the Holocene has an astronomical origin linked to a major recurrence of particular displacements of the planets around the Sun. In the choice of the 
appropriate astronomical proxy, we were inspired by the Milankovic $(1930)$ theory that links the $\sim 100,000$ yr variation of the Earth's orbit eccentricity to the glacial cycles of the past 1 million years. Thus, we hypothesize that the Hallstatt cycle could derive from an expansion-contraction rhythmic dynamics of the solar system driven by the rotation of the planets that yields to a specific set of stable orbital resonances.

The dynamics of the solar system circularity is well described by the wobbling of the planetary mass center (PMC) orbiting the Sun, which scales the wobbling of the Sun relative to the barycenter of the solar system. We used the ephemeris of the Sun relative to the barycenter to derive such a complex orbit. Then we used a proposed Keplerian constant of motion, the eccentricity vector (e.g.: Mungan, 2005), to evaluate how the instantaneous eccentricity of the orbit of the PMC varies in time. Using this observable we demonstrate that the solar system circularity pulses with a 2100-2500 period together with a number of already known oscillations associated to the orbital periods of the planets.

The situation of having several stable orbital resonances and orbital proxies made of many harmonics is not surprising because of the complexity of the solar system. It is, however, highly confusing for identifying the possible physical origin of a specific oscillation. The evident analogous is the theory of ocean tides where generic tidal generation potentials deduced from the Sun's and Moon's orbits relative to the Earth produce a very large number of tidal constituent waves (Doodson, 1921, Melchior, 1978). These oscillations are differentiated in the literature with a very long list of Darwin's symbols indicating their physical origin such as the $N$ (lunar Saros) tidal wave, $S a$ (solar annual) tidal wave, $M m$ (lunar monthly) tidal wave, $M 2$ (principal lunar semidiurnal) tidal wave, the $S 2$ (principal solar semidiurnal) tidal wave, $N 2$ (larger lunar elliptical semidiurnal) tidal wave, etc (Darwin, 1902). Alternative and specific astronomical proxies are needed to well highlight each of these oscillations because they have a different physical origin and many of them are just small perturbations of the dominant $M 2$ and $S 2$ waves.

To better identify the physical origin of the astronomical 2100-2500 yr cycle, and to separate it from the fast and larger oscillations associated to the orbits of the planets, we searched for more appropriate astronomical proxies. We collected the times it takes the Sun to make pericycles and apocycles and the times it takes the Sun to move from its minimum to maximum distances from the barycenter within these arcs. We show that these particular astronomical proxies reveal a macroscopic 2100-2500 yr period oscillation perfectly coherent to the Hallstatt oscillation found in the radionucleotide records with a statistical confidence above $95 \%$.

Finally, we briefly hypothesize the physical mechanisms involved in the process suggesting that the pulses of the solar system could be modulating the solar wind and by that the incoming cosmic ray flux and the cosmic dust concentration surrounding the Earth (cf: Mörner, 1996).

\section{The 2100-2500 yr Hallstatt cycle in the $\Delta^{14} C$ nucleotide record}

Figure 1A shows the $\Delta^{14} C(\%)$ record (IntCal04.14c) from 10,500 B. C. to $1845 \mathrm{~A}$. D. that covers the entire Holocene (data are from Reimer et al., 2004). This record was obtained using dendrochronological dating and cross-checking from several millennia-long tree-ring chronologies. The IntCal04.14c record extends for 26,000 years. The last 12,500 years are reported with a 5-year resolution and the data derive mostly from tree-ring chronologies. For older dates this record is made using mostly marine (e.g. coral) records that have a lower resolution. The second component of the record, the one obtained mostly from marine chronologies, is not used here because it shows altered and very smooth patterns compared to the tree-ring chronologies. This difference is very likely due to the diverse physical properties of the two sets as one uses trees that absorb carbon directly from the air where ${ }^{14} C$ is produced by cosmic rays, while the other uses corals that absorb carbon dissolved in the water where it can remain diluted for very long times. 

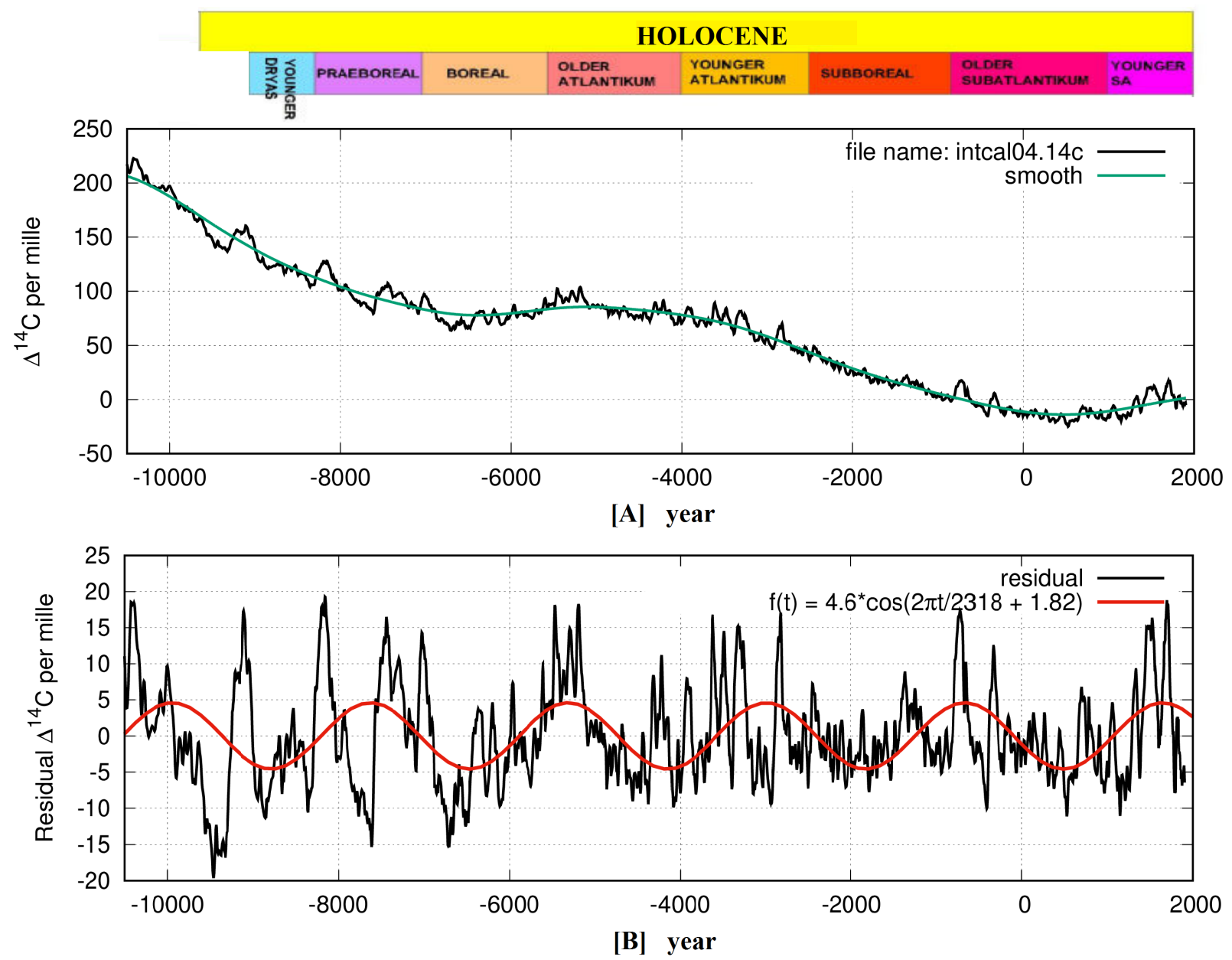

Figure 1: $[\mathrm{A}] \Delta^{14} C(\%)$ record (black) throughout the Holocene from -10500 B. C. to 1900 A. D. and its multi-millennial smooth curve (green). [B] A residual signal obtained by detrending the smooth curve from the data. The latter is a fit with a sinusoidal function (red). This signal covers the period from 10500 B. C. to 1900 A. D. The figures report the name of the original file downloaded from https://www.radiocarbon.org/IntCal04.htm

Figure 1A also shows in green a gnuplot acsplines smooth curve that captures the multi-millennial modulation of the record that appears to be characterized by a trend plus an approximate 6,000-7,000 year modulation, which is observed throughout the entire 26,000 years of the original record. The physical origin of the trend and this long oscillation are not discussed herein. Figure 1B shows the residual signal obtained by detrending the smooth curve from the original data from 10,500 B. C. to 1900 A. D.. Note that since 1900 the radionucleotide record has been likely contaminated from increasing anthropogenic $\mathrm{CO}_{2}$ emissions and since 1950 because of atmospheric nuclear bomb tests.

The residual record depicted in Figure 1B is relatively similar to the data made available in file Resid04_2000.14c (Reimer et al. 2004) where a smoothing spline approximating a 2000-year moving average was used (cf: Stuiver et al. 1998). However, our adopted filtering is significantly smoother and better preserves the patterns at scales shorter than 5000 years. This operation avoids artifacts that might interfere with the Hallstatt cycle.

The residual record presents two major oscillations at about 900-1050 yr period (the Eddy cycle) and at 2100-2500 year period (the Hallstatt cycle): see the periodogram in Figure 10. The quasi millennial 
oscillation found in nucleotide record has been extensively studied in the literature. It was found coherent with a quasi-millennial climate large oscillation (e.g.: Bond et al., 2001, Kerr, 2001) and was reconstructed with a combination of Jupiter-Saturn tidal induced oscillations and the 11-year sun-spot oscillation (e.g.: Scafetta, 2012b, 2014a). Herein, we will not focus on this oscillation either, but only on the longer Hallstatt cycle.

We attempted to fit the residual record depicted in Figure 1B with a harmonic curve of the type

$$
f(t)=A \cos (2 \pi t / T+\phi)+C
$$

However, the statistical fit gives slightly different values according to the chosen time interval. For example: from $6000 \mathrm{~B}$. C. to $1900 \mathrm{~A}$. D. it gives $T=2357 \pm 24 \mathrm{yr}$; from 7,000 B. C. to $1,900 \mathrm{~A}$. D. it gives $T=2326 \pm 18$ yr; from 8,000 B. C. to 1,900 A. D. it gives $T=2249 \pm 16 \mathrm{yr}$; from 9,000 B. C. to 1,900 A. D. it gives $T=2311 \pm 15 \mathrm{yr}$; and from $10,500 \mathrm{~B}$. C. to $1,900 \mathrm{~A}$. D. it gives $T=2402 \pm 14 \mathrm{yr}$. The harmonic function fit also gives a slightly variable phase according to the fit interval of about $\phi=2 \pm 0.2$.

Such a variable result is not surprising in analyzing experimental geophysical, radionucleotide or paleoclimatic records spanning several thousands years since these records are characterized by some uncertainty both in the amplitude and in the timing of the data. Moreover, the records may be influenced by different physical sources that could induce a certain pattern variability. In any case, since the statistical error of the periodogram associated to a spectral peak period is $\nabla p= \pm p^{2} / 2 L$, where $L=12,500$ yr is the length of the record shown in Figure 1B, an observed spectral peak period at the 2100-2500 yr time scale is characterized by a spectral error of about is $\pm 200 \mathrm{yr}$, which well covers the typical oscillation range found in radionucleotide $\left({ }^{14} \mathrm{C}\right.$ and $\left.{ }^{10} \mathrm{Be}\right)$ records regarding the Hallstatt cycle, as reported in the scientific literature.

Given the above uncertainty, in Figure 1B we fit the record with an harmonic function with period $T=2318 \mathrm{yr}$ for the reasons explained in Section 3 and also because such period is nearly recovered by the fit value from $9,000 \mathrm{~B}$. C. to 1,900 A. D. that covers the Holocene after the end of the last glacial period.

The harmonic function depicted in Figure 1B uses the fit parameters obtained during the period between 750 B. C. and 1750 A. D., which covers the first Hallstatt oscillation observed in the radionucleotide record. During this period the data are likely the most accurate of the record because the uncertainty increases with time. The phase shift of the harmonic function is $\phi=1.82$. Thus, the maximum of the radiocarbon Hallstatt cycle corresponds to about 1645 A. D., namely the beginning of the Maunder solar Minimum [1645-1715], which was the most significant solar minimum of the last millennium.

As shown in Figure 1B, the depicted harmonic function well predicts the previous Hallstatt maxima in the radionucleotide record that corresponded to the coldest epochs of the Holocene occurred around these periods: 10,000 B. C. (Younger Dryas cooling onset), 7,500 B. C. (Early Holocene cooling event), 5,300 B. C. (Boreal/Atlantic transition and precipitation change), 3,000 B. C. (Mid-Holocene Transition.), 700 B. C. (Sub-Atlantic Minimum that also yielded the Greek Dark Ages). Indeed, O'Brien et al. (1995) found that also the polar atmospheric circulation changes are regulated by a Hallstatt oscillation throughout the Holocene. A cooling-warming cycle of about 2100-2500 year is, indeed, observed throughout the Holocene in numerous climate records (e.g.: Bond et al., 2001, Marcott et al., 2013, Mayewski et al., 2004). Also a quasi millennial cycle is observed in climate and radionucleotide records (cf.: Bond et al., 2001, Kerr, 2001, Scafetta, 2013, 2014a).

In any case, note that the adoption of a fit phase of about $\phi=2$ would induce a temporal shift of about 65 years relative to the chosen harmonic depicted in Figure 1B, and the maximum of the 2318 year cycle would fall in 1580 A. D., which is between the Spörer solar Minimum [1450-1550] and the Maunder Minimum [1645-1715]. Thus, in any case, the strong solar minimum of the 16th-17th centuries was likely driven by the Hallstatt cycle. For the purpose of this paper the difference between the two phases is nearly negligible. 


\section{The Jupiter-Saturn-Uranus-Neptune 2318-year stable resonance}

An important concept in celestial mechanics is that of orbital resonance. A resonance occurs when two or more orbiting bodies can exert a regular, periodic gravitational influence on each other. This happens when their orbital periods are related by a ratio of small integers. Orbital resonances greatly enhance the mutual gravitational influence of the bodies and, therefore, of the space symmetry of an orbiting system such as the heliosphere of the solar system. Well known examples of orbital resonances are the 1:2:4 resonance of Jupiter's moons Ganymede, Europa and Io, the 2:3 resonance between Pluto and Neptune, the various resonances that regulate the asteroid belt etc.. Indeed, the entire solar system appears to be synchronized by specific orbital resonances (cf. Scafetta, 2014b; Tattersall, 2013a). Thus, we hypothesize that the Hallstatt oscillation found in radionucleotide and climatic records could be the result of a specific orbital resonance within the solar system. All planets could be involved in the process but, because of the length of the Hallstatt oscillation, it is more reasonable to search a resonance that links the four Jovian planets: Jupiter, Saturn, Uranus and Neptune.

A system of periods $T_{i}$ is said to be in linear resonant state if there exists a set of small integer numbers $a_{i}$ such that:

$$
\frac{1}{T}=\left|\sum \frac{a_{i}}{T_{i}}\right|<\gamma
$$

where $i=1, \ldots, N$. N is the number of orbiting objects, $\gamma$ a very small number and $T$ the resonance period. The simplest case of resonance is when two orbital periods (e.g. $P_{1}$ and $P_{2}$ ) have an integer ratio: $P_{1} / P_{2}=n$, where $\mathrm{n}$ is the integer 1,2 or 3 etc.

A linear resonance is also stable if its resonance order is zero, that is if $\sum a_{i}=0$. Stable resonances are independent on the selection of the rotating reference system. In fact, relative to any observer moving with any period $M$ with regard to stars, each orbital body would rotate with a frequency $f_{i M}=1 / T_{i}-1 / M$. It is easy to demonstrate that for stable resonances it holds: $T^{-1}=\sum a_{i} / T_{i}=\sum a_{i} f_{i M}$. Therefore, stable resonances significantly characterize the physical properties of an orbital system.

The simplest cases of stable resonances are the synodical periods between two orbiting objects whose frequency is given by $f_{12}=\left|1 / P_{1}-1 / P_{2}\right|$. For example: the synodic or conjunction period between Jupiter $\left(P_{J}=4332.589\right.$ days $)$ and Saturn $\left(P_{S}=10759.22\right.$ days $)$ is $P_{J S}=\left(P_{J}^{-1}-P_{S}^{-1}\right)^{-1}=7253.455$ days $=19.859$ years; the synodic or conjunction period between Uranus $\left(P_{U}=30685.4\right.$ days $)$ and Neptune $\left(P_{N}=60189.0\right.$ days) is $P_{U N}=\left(P_{U}^{-1}-P_{N}^{-1}\right)^{-1}=62599.94$ days $=171.393$ years. The orbital periods are from NASA (http://nssdc.gsfc.nasa.gov/planetary/factsheet/); we use the conversion: 1 year $=365.2425$ days.

There are very few stable orbital resonances and if the coefficients $a_{i}$, which must be small, are chosen between -3 and 3 only one resonance falls within the Hallstatt time scale of 2100-2500 year and, in general, for periods larger than 200 years. This is a combination of the orbital periods of Jupiter, Saturn, Uranus and Neptune such that:

$$
f_{J S U N}=\frac{1}{P_{j}}-\frac{3}{P_{S}}+\frac{1}{P_{U}}+\frac{1}{P_{N}} .
$$

The period of such a resonance is

$$
P_{J S U N}=\frac{1}{f_{J S U N}}=846471.447 \mathrm{~d}=2317.56 \mathrm{yr} .
$$

Since the above resonance is stable, the same period can be determined by any observer moving with any period $\mathrm{M}$ with regard to stars. The physical meaning of the above resonance will be demonstrated in the following sections. Herein we stress that this resonance involves a combination of all four Jovian planets. We 
also notice that such a resonance nearly corresponds to about 116.5 revolutions of the conjunction period of Jupiter and Saturn $(116.5 * 19.859=2313.6 \mathrm{yr})$, and 13.5 revolutions of the conjunction period of Uranus and Neptune $(13.5 * 171.393=2313.8 \mathrm{yr})$. Thus, every about 2313.7 years there exists a repeating pattern involving conjunctions and oppositions among the four Jovian planets of the solar system whose gravitational effect is revealed in the following sections.

Additional resonances can be calculated by making the coefficients $a_{i}$ to vary within a larger range. However, even if this range is chosen to be between -10 and 10, the only stable resonance periods found for periods larger than 1000 years are at $1158.78 \mathrm{yr}$ (resonance 2:-6:2:2), 1159.30 yr (resonance -1:2:4:-5), 2317.56 yr (resonance 1:-3:1:1), 2319.62 yr (resonance -2:5:3:-6) and 2,607,251.87 yr (resonance 3:-8:-2:7). Thus, the period of 2317-2320 years represents a very important and unique stable resonance of the solar system. We note that Humlum et al. (2011) found a 1139 $\pm 160 \mathrm{yr}$ oscillation in the detrended GISP2 surface temperature series during the last 4000 years and Davis and Bohling (2001) found a spectral peak between $950 \mathrm{yr}$ and $1113 \mathrm{yr}$, which may be coherent to the above millennial stable resonances although for the Eddy cycle there could be alternative explanations (e.g.: Scafetta, 2012a, 2014a).

Two other important stable resonances that we will meet in the next sections are:

$$
f_{J S U}=\frac{1}{P_{J}}-\frac{3}{P_{S}}+\frac{2}{P_{U}}
$$

which gives $P_{J S U}=159.59 \mathrm{yr}$ and

$$
f_{J S N}=-\frac{1}{P_{J}}+\frac{3}{P_{S}}-\frac{2}{P_{N}}
$$

which gives $P_{J S N}=185.08 \mathrm{yr}$.

We will demonstrate that, using opportune astronomical observables, the 2318-year resonance appears like a modulation of these faster resonance oscillations together with that of the Uranus-Neptune synodic stable resonance $P_{U N}=171.393$ yr. The three 159-171-185 yr astronomical resonances are very important also for supporting the main hypothesis of our paper, namely to interpret astronomically the origin of the Hallstatt oscillation observed in radionucleotide and climate records. In fact, these resonances, in particular $P_{U N}$, have already been found to characterize such geophysical records used to reconstruct also cosmic ray flux and solar activity throughout the Holocene (cf.: McCracken et al., 2014, Sharp, 2013) and also with aurora records available since the 16th century (Scafetta and Willson, 2013a).

Table 1 reports a list of stable resonances for periods larger than 5 years associated to the Jupiter-SaturnUranus-Neptune system where the coefficients $a_{i}$ are made to vary between -3 and 3 . These resonances are clustered around specific frequencies. In particular, note the resonance clusters at 44-46 years, 57-62 years and 82-97 years that are found in solar and aurora activity (e.g.: McCracken et a., 2001; Ogurtsov et al. 2002 scafetta and Willson, 2013a; Scafetta, 2014a, Vaquero et al., 2002) and also in climate records (e.g.: Czymzik et al. 2016, Hoyt and Schatten, 1997, and many others), e.g. a quasi 60-year cycle is very important in climate (e.g.: Gervais, 2016, Loehle and Scafetta, 2011; Manzi et al., 2012, Mazzarella and Scafetta, 2012, Scafetta, 2010, 2014c; Wyatt and Curry, 2014, and many others). The 82-97 year period is known as the Gleissberg cycle.

In the next section we will construct physical observables that better reveal the above Jupiter-SaturnUranus-Neptune resonances. From a purely spectral point of view, it may be pointed out that many functions of the orbits of the planets (e.g. total angular momentum of the planets, speed and position of the PMC relative to the Sun, etc) are expected to present numerous common spectral peaks simply because the harmonic input would be the same. We will use the eccentricity function of the Sun-PMC orbit and other specific orbital proxies because these proxies suggest a possible physical mechanism, as we will discuss in the 


\begin{tabular}{|c|c|c|c|c|c|c|c|c|c|}
\hline$a_{\text {Jup }}$ & $a_{\text {Sat }}$ & $a_{U r a}$ & $a_{N e p}$ & $\mathrm{~T}(\mathrm{yr})$ & $a_{\text {Jup }}$ & $a_{\text {Sat }}$ & $a_{U r a}$ & $a_{N e p}$ & $\mathrm{~T}(\mathrm{yr})$ \\
\hline 3 & $2-1$ & -2 & 0 & 5.12 & 2 & $2-2$ & $2-2$ & 2 & $\begin{array}{ll}11.23 \\
\end{array}$ \\
\hline 2 & 2 & -3 & -1 & 5.14 & 1 & 1 & -3 & 1 & 11.29 \\
\hline 3 & -2 & 2 & -3 & 5.25 & 2 & -3 & 2 & -1 & 11.83 \\
\hline 3 & -1 & -3 & 1 & 5.28 & 1 & 0 & 1 & -2 & 11.90 \\
\hline 3 & -2 & 1 & -2 & 5.41 & 0 & 3 & 0 & -3 & 11.96 \\
\hline 2 & 1 & 0 & -3 & 5.42 & 2 & -2 & -3 & 3 & 12.02 \\
\hline 3 & -2 & 0 & -1 & 5.59 & 2 & -3 & 1 & 0 & 12.71 \\
\hline 2 & 1 & -1 & -2 & 5.60 & 1 & 0 & 0 & -1 & 12.78 \\
\hline 3 & -2 & -1 & 0 & 5.78 & 0 & 3 & -1 & -2 & 12.85 \\
\hline 2 & 1 & -2 & -1 & 5.79 & 2 & -3 & 0 & 1 & 13.73 \\
\hline 3 & -3 & 3 & -3 & 5.93 & 1 & 0 & -1 & 0 & 13.81 \\
\hline 3 & -2 & -2 & 1 & 5.98 & 0 & 3 & -2 & -1 & 13.90 \\
\hline 2 & 1 & -3 & 0 & 5.99 & 1 & -1 & 3 & -3 & 14.74 \\
\hline 3 & -3 & 2 & -2 & 6.15 & 2 & -3 & -1 & 2 & 14.93 \\
\hline 2 & 0 & 1 & -3 & 6.16 & 1 & 0 & -2 & 1 & 15.02 \\
\hline 3 & -2 & -3 & 2 & 6.19 & 0 & 3 & -3 & 0 & 15.12 \\
\hline 3 & -3 & 1 & -1 & 6.37 & 1 & -1 & 2 & -2 & 16.12 \\
\hline 2 & 0 & 0 & -2 & 6.39 & 0 & 2 & 1 & -3 & 16.24 \\
\hline 1 & 3 & -1 & -3 & 6.41 & 2 & -3 & -2 & 3 & 16.35 \\
\hline 3 & -3 & 0 & 0 & 6.62 & 1 & 0 & -3 & 2 & 16.47 \\
\hline 2 & 0 & -1 & -1 & 6.64 & 1 & -1 & 1 & -1 & 17.80 \\
\hline 1 & 3 & -2 & -2 & 6.66 & 0 & 2 & 0 & -2 & 17.93 \\
\hline 3 & -3 & -1 & $\overline{1}$ & 6.89 & 1 & -1 & 0 & 0 & 19.86 \\
\hline 2 & 0 & -2 & 0 & 6.91 & 0 & 2 & -1 & -1 & 20.03 \\
\hline 1 & 3 & -3 & -1 & 6.93 & 1 & -1 & -1 & 1 & 22.46 \\
\hline 2 & -1 & 2 & -3 & 7.13 & 0 & 2 & -2 & 0 & 22.68 \\
\hline 3 & -3 & -2 & 2 & 7.17 & 1 & -2 & 3 & -2 & 25.01 \\
\hline 2 & 0 & -3 & 1 & 7.20 & 0 & 1 & 2 & -3 & 25.29 \\
\hline 2 & -1 & 1 & -2 & 7.44 & 1 & -1 & -2 & 2 & 25.85 \\
\hline 1 & 2 & 0 & -3 & 7.46 & 0 & 2 & -3 & 1 & 26.14 \\
\hline 3 & -3 & -3 & 3 & 7.49 & 1 & -2 & 2 & -1 & 29.29 \\
\hline 2 & -1 & 0 & -1 & 7.78 & 0 & 1 & 1 & -2 & 29.66 \\
\hline 1 & 2 & -1 & -2 & 7.80 & 1 & -1 & -3 & 3 & 30.44 \\
\hline 2 & -1 & -1 & 0 & 8.15 & 1 & -2 & 1 & 0 & 35.32 \\
\hline 1 & 2 & -2 & -1 & 8.18 & 0 & 1 & 0 & -1 & 35.87 \\
\hline 2 & -2 & 3 & -3 & 8.46 & 1 & -2 & 0 & 1 & 44.49 \\
\hline 2 & -1 & -2 & 1 & 8.55 & 0 & 1 & -1 & 0 & 45.36 \\
\hline 1 & 2 & -3 & 0 & 8.58 & 0 & 0 & 3 & -3 & 57.13 \\
\hline 2 & -2 & 2 & -2 & 8.90 & 1 & -2 & -1 & 2 & 60.09 \\
\hline 1 & 1 & 1 & -3 & 8.93 & 0 & 1 & -2 & 1 & 61.69 \\
\hline 2 & -1 & -3 & 2 & 9.00 & 1 & -3 & 3 & -1 & 82.64 \\
\hline 2 & -2 & 1 & -1 & 9.39 & 0 & 0 & 2 & -2 & 85.70 \\
\hline 1 & 1 & 0 & -2 & 9.42 & -1 & 3 & 1 & -3 & 88.99 \\
\hline 2 & -2 & 0 & 0 & 9.93 & 1 & -2 & -2 & 3 & 92.54 \\
\hline 1 & 1 & -1 & -1 & 9.97 & 0 & 1 & -3 & 2 & 96.39 \\
\hline 2 & -2 & -1 & 1 & 10.54 & 1 & -3 & 2 & 0 & 159.59 \\
\hline 1 & 1 & -2 & 0 & 10.59 & 0 & 0 & 1 & -1 & 171.39 \\
\hline 2 & -3 & 3 & -2 & 11.07 & -1 & 3 & 0 & -2 & 185.08 \\
\hline 1 & 0 & 2 & -3 & 11.12 & 1 & -3 & 1 & 1 & 2317.56 \\
\hline
\end{tabular}

Table 1: Stable resonances associated to the Jupiter-Saturn-Uranus-Neptune system. The coefficients $a_{i}$ of Eq. 2 are made to vary between -3 and 3 . See also Figure 4B. 
next sections.

\section{The eccentricity vector}

Let us fully derive the instantaneous eccentricity function of the orbit of a generic planet orbiting the Sun (cf: Mungan, 2005). In classical celestial mechanics a Keplerian orbit is defined as the motion of an object orbiting another (e.g. a planet orbiting its star) under Newton's force of gravity:

$$
m \boldsymbol{a}=-\frac{G M m}{r^{2}} \hat{\boldsymbol{r}}
$$

Because $\boldsymbol{r}=r \hat{\boldsymbol{r}}$ and the angular momentum $\boldsymbol{L}=m \boldsymbol{r} \times \boldsymbol{v}$ is constant, Eq. 7 can be easily rewritten as

$$
\frac{d}{d t}(\boldsymbol{v} \times \boldsymbol{L})=G M m \frac{d \hat{\boldsymbol{r}}}{d t},
$$

where $\boldsymbol{v}=d \boldsymbol{r} / d t$ is the velocity of the orbiting body. By integrating Eq. 8 , we obtain

$$
\boldsymbol{v} \times \boldsymbol{L}=G M m(\hat{\boldsymbol{r}}+\boldsymbol{e}),
$$

where $\boldsymbol{e}$ is an integration constant vector. After a simple vector algebra we obtain

$$
\boldsymbol{e}=\frac{\boldsymbol{v} \times(\boldsymbol{r} \times \boldsymbol{v})}{G M}-\hat{\boldsymbol{r}}
$$

To understand the physical meaning of the vector $\boldsymbol{e}$, we take the dot product of the position vector $\boldsymbol{r}$ with Eq. 9 to obtain

$$
\boldsymbol{r} \cdot(\boldsymbol{v} \times \boldsymbol{L})=G M m \boldsymbol{r} \cdot(\hat{\boldsymbol{r}}+\boldsymbol{e})
$$

that becomes

$$
m(\boldsymbol{r} \times \boldsymbol{v}) \cdot \boldsymbol{L}=L^{2}=G M m^{2}(1+e \cos \theta) r .
$$

If $c=L^{2} /\left(G M m^{2}\right)$ and the vector $\boldsymbol{e}$ is chosen to point towards the periapsis of the orbit, in the traditional $r-\theta$ polar coordinates, Eq. 12 is equivalent to the traditional Keplerian orbital equation:

$$
r(\theta)=\frac{c}{1+e \cos \theta},
$$

where $c$ is a constant called the semi-latus rectum of the curve and $e$ is the eccentricity of the orbit.

For a circular orbit $e=0$; for an elliptical orbit $0<e<1$ and $\theta=0$ and $\theta=\pi$ indicate the position of the perihelion and aphelion, respectively; for a parabolic trajectory $e=1$; and for a hyperbolic trajectory $e>1$. In the case of a simple two-body system, without any form of dissipation or perturbation, celestial mechanics predicts that the eccentricity $e$ of an orbit is constant. Thus, Eq. 10 defines the eccentricity vector, whose scalar is the eccentricity of the Keplerian orbit.

Using simple vector algebra, $\boldsymbol{v} \times(\boldsymbol{r} \times \boldsymbol{v})=(\boldsymbol{v} \cdot \boldsymbol{v}) \boldsymbol{r}-(\boldsymbol{r} \cdot \boldsymbol{v}) \boldsymbol{v}$, Eq. 10 can be rewritten in a more friendly way, and the instantaneous eccentricity of the trajectory of each planet of the solar system can be defined as

$$
e=\left|\left(\frac{v^{2}}{\mu}-\frac{1}{r}\right) \boldsymbol{r}-\frac{\boldsymbol{r} \cdot \boldsymbol{v}}{\mu} \boldsymbol{v}\right|,
$$

where $\mu=G M_{\text {sun }}=2.959122082855911 \cdot 10^{-4} A U^{3} / d^{2}$ is the standard gravitational parameter for the Sun as used in the adopted ephemeris files (Folkner et al., 2014. Table 8). However, Eq. 7 works if $M \gg m$. In 
real cases, the mass $m$ on the left side of 7 must be substituted with the reduced mass, $M_{\text {sun }} m_{\text {planet }} /\left(M_{\text {sun }}+\right.$ $\left.m_{\text {planet }}\right)$, which yields again to Eq. 14 with the following correction $\mu=G\left(M_{\text {sun }}+m_{\text {planet }}\right)$, as we will use in the following section.

\section{Definition of the planetary mass center relative to the Sun}

The wobbling of the Sun occurs mostly close to the ecliptic orbital plane and it is a real feature of the solar system relative from the outer deep space from where the cosmic ray flux comes. We hypothesized that the rhythmic contraction and expansion of the solar system disk associated to its inner wobbling could modulate the incoming cosmic ray flux reaching the Earth and/or alter the physical properties of the heliosphere modulating the solar wind and interplanetary/cosmic dust concentration. This dynamics can be represented by the movement of the planetary mass center (PMC) relative to the Sun. This orbit is deduced in the following way using programs that implement the ephemeris files DE431/DE432 prepared by the NASA Solar System Dynamics Group of the Jet Propulsion Laboratory (ftp://ssd.jpl.nasa.gov/pub/eph/planets/ascii) (Folkner et al., 2014; Folkner, 2014).

Folkner et al. (2014) reports that the orientation of the DE431/DE432 ephemeris is tied to the International Celestial Reference Frame with an accuracy of 0.0002": for the inner planets the orbital accuracy is of the order of a few hundred meters, for Jupiter and Saturn the orbital accuracy is of tens of kilometers and for Uranus, Neptune, and Pluto the orbital accuracy worsen up to several thousand kilometers. This means that the orbital parameters have at least a 9 to 7 digit precision from the inner planets up to Pluto, respectively. Thus, the ephemeris error-bars alone are not expected to provide false evidences for major cycles, also because several of the observed spectral peaks of the adopted astronomical observables can be easily recognized as stable orbital resonances or orbital periods, as shown below.

Let $\boldsymbol{r}_{p}$ be the vector position of the center of mass of all objects of the solar system, Sun excluded, relative to the barycenter; let $\boldsymbol{r}_{s}$ be the vector position of the Sun relative to barycenter; let $M_{p}$ be the total mass of all objects of the solar system excluded the Sun, that is the sum of the 352 masses (planets + asteroids) taken into account by the DE432 JPL ephemeris file; let $M_{s}$ be the mass of the Sun. These masses are deduced from the parameters of the header file of the NASA JPL DE432 ephemeris file.

Relative to the barycenter, the position vectors and the relative velocities are balanced, that is, observing that $M_{p} \boldsymbol{r}_{p}=\sum_{i} m_{i} \boldsymbol{r}_{i}$ and $M_{p} \boldsymbol{v}_{p}=\sum_{i} m_{i} \boldsymbol{v}_{i}$ where the index i refers to each planetary or asteroid object of the solar system, the following equations are fulfilled:

$$
\begin{aligned}
& M_{s} \boldsymbol{r}_{s}+M_{p} \boldsymbol{r}_{p}=0 \\
& M_{s} \boldsymbol{v}_{s}+M_{p} \boldsymbol{v}_{p}=0 .
\end{aligned}
$$

Let $\boldsymbol{r}$ and $\boldsymbol{v}$ be the position and velocity vector, respectively, of the PMC relative to the the Sun. Thus, we have:

$$
\begin{gathered}
\boldsymbol{r}=-\boldsymbol{r}_{s}+\boldsymbol{r}_{p}=\frac{M_{s}+M_{p}}{M_{p}}\left(-\boldsymbol{r}_{s}\right) \\
\boldsymbol{v}=-\boldsymbol{v}_{s}+\boldsymbol{v}_{p}=\frac{M_{s}+M_{p}}{M_{p}}\left(-\boldsymbol{v}_{s}\right)
\end{gathered}
$$

Figure 2 shows a section of the orbit of PMC from 1950 to 2050 where it is seen that the PMC point wobbles around the Sun within an orbit of about 7 AU radius. 


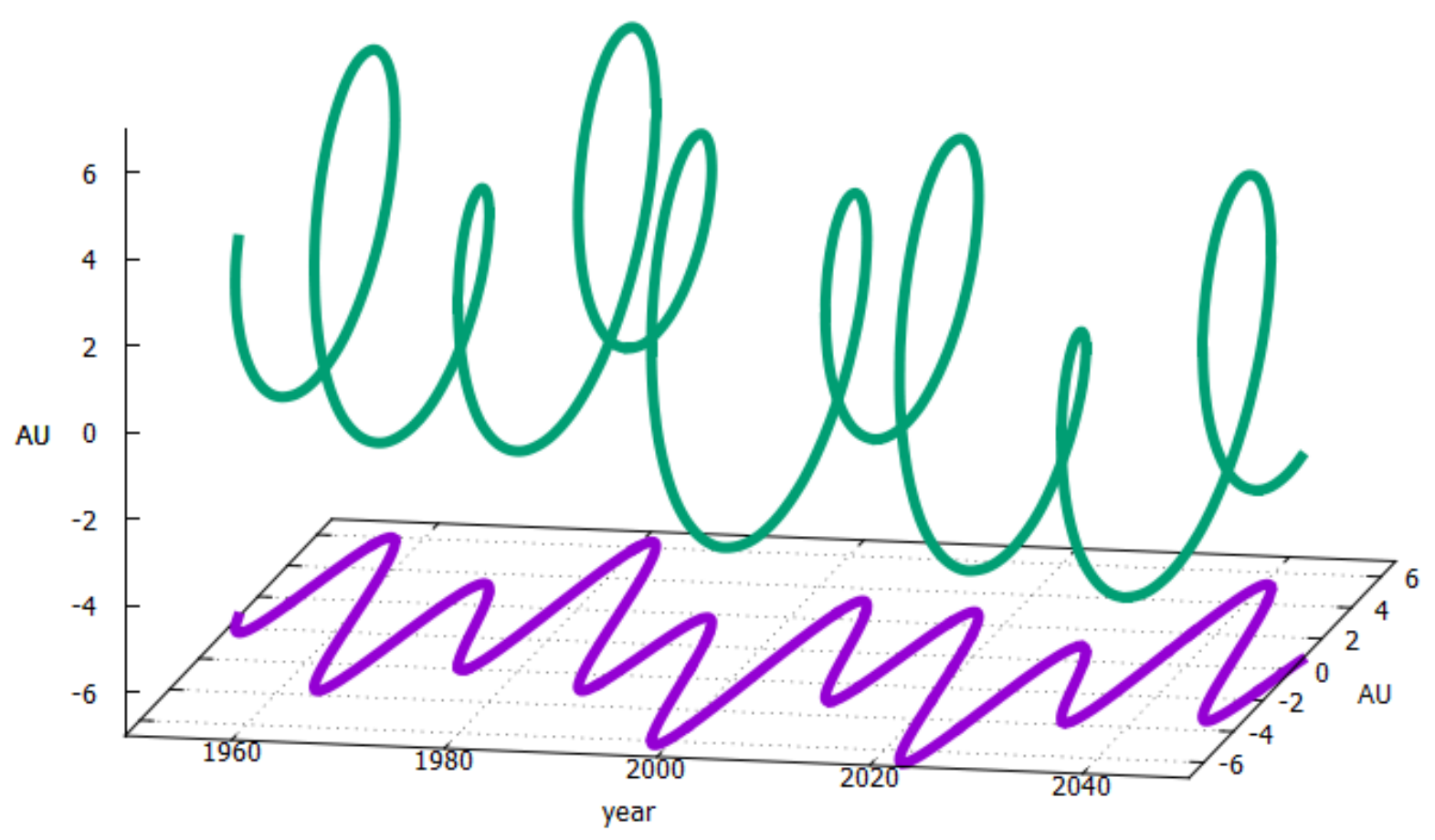

Figure 2: Motion of the PMC relative to the Sun from 1950 to 2050.

Although Eq. 14 can be rigorously applied only to a Keplerian orbit, and is useful to determine for example the small fluctuations of the orbits of the planets of the solar system, we can assume that the PMC, which does not follow a Keplerian orbit, at each instant represents a given planet $\mathrm{P}$ that is orbiting the Sun at that specific position $\boldsymbol{r}$ and with that specific velocity $\boldsymbol{v}$ estimated in Eqs. 17 and 18 Then, we define the instantaneous eccentricity of the orbit of PMC as the eccentricity of the hypothetical orbit of the planet $\mathrm{P}$ evolving in time using Eq. 14, the $\boldsymbol{r}$ and $\boldsymbol{v}$ vectors estimated in Eqs. 17 and 18 and $\mu=G\left(M_{s}+M_{C M P}\right)=2.963092749817812 \cdot 10^{-4} A U^{3} / d^{2}$, which takes into account all masses of the solar system used in the ephemeris files DE431/DE432.

\section{Analysis of the "eccentricity" variation of the Sun-PMC orbit}

Figure 3 shows the record of the eccentricity $e$ of the Sun-PMC orbit calculated by Eq. 14 from 13,000 B. C. to 17,000 A. D. sampled every 30 days. Figure 4A shows its periodogram and Figure 4B compares it with the periods of the stable planetary resonances reported in Table 1. A clear correspondence is found between all spectral peaks of the eccentricity function of the Sun-PMC orbit and the stable resonances generated by Jupiter, Saturn, Uranus and Neptune.

As expected, the instantaneous eccentricity of the Sun-PMC orbit varies greatly from a trajectory nearly circular $(e \approx 0)$ to one nearly parabolic $(e \approx 1)$. Large oscillations are observed, in particular at the 19.86 yr synodic period between Jupiter and Saturn. Other strong oscillations close to the known planetary orbital periods of the four Jovian planets are observed: Jupiter, $11.86 \mathrm{yr}$; Saturn, $29.46 \mathrm{yr}$; Uranus, 84.01 yr; Neptune, 164.8 yr. In addition, several other synodic periods among planets are observed as well: Earth-Jupiter, 1.092 yr; Jupiter-Uranus, 13.8 yr; Jupiter-Neptune, 12.78 yr; Saturn-Uranus, 45.4 yr; SaturnNeptune, 38.9 yr; Uranus-Neptune, 172 yr. Also the Jupiter-Saturn trigon synodic period, 57-61 yr, is well observed. All these known oscillations were well expected. 


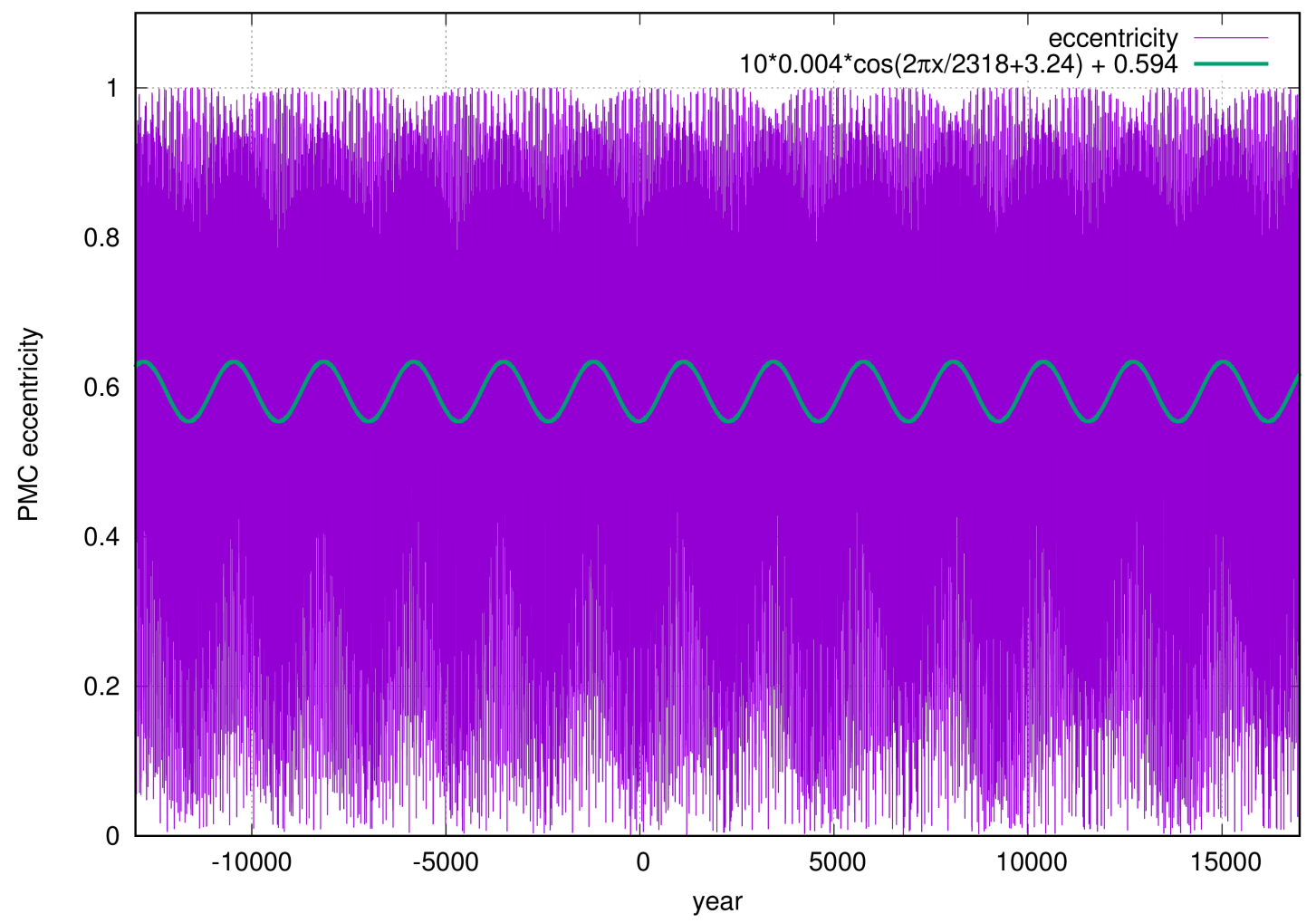

Figure 3: Variation of the eccentricity (Eq. 14) of the PMC relative to the Sun. The latter is fit with a sinusoidal function (green) whose amplitude has been magnified by 10 for visual convenience.

For what concerns this study, Figures 3 and 4 also demonstrate that the chosen eccentricity function presents a major oscillation at about 2100-2500 yr period that could not be immediately derived from the individual planetary orbital periods. The statistical error of the periodogram associated to a spectral peak period is $\nabla p= \pm p^{2} / 2 L$, where $L=30000 \mathrm{yr}$ is the length of the record analyzed. The observed periodogram peak period is at $p=2318 \pm 90 \mathrm{yr}$. Thus, it is evidently due to the Jupiter-Saturn-UranusNeptune resonance discussed in section 3 . This periodicity is perfectly coherent with the Hallstatt oscillation found in the radionucleotide records as that shown in Figures 1: see Figure 10. The periodogram depicted in Figure 4 stresses that the 2318 yr period peak is the most relevant within the spectral range between 200 and $10000 \mathrm{yr}$ periods indicating that this oscillation dominates this time scale, as also found for the stable resonances reported in Table 1 .

Figure 5 compares the cosine curves used to fit both the radionucleotide record depicted in Figure 1B, and the eccentricity function depicted in Figure 3. The periods are the same, within their error of measure, and the phases are $\phi \approx 1.82$ and $\phi \approx 3.24$, respectively. Thus, as Figure 5 (upper panel) shows, the two harmonics are shifted by almost $\pi / 2$ or about 525 years. This means that the Hallstatt oscillation of the radionucleotide record is nearly proportional to the integration or to the negative of the derivative of the eccentricity record of the Sun-PMC orbit.

Figure 5 shows that, on the Hallstatt-cycle time scale, a larger production of radionucleotide particles occurs while the Sun-PMC orbit evolves from statistically more elliptical shapes $(e \approx 0.598)$ to statistically 


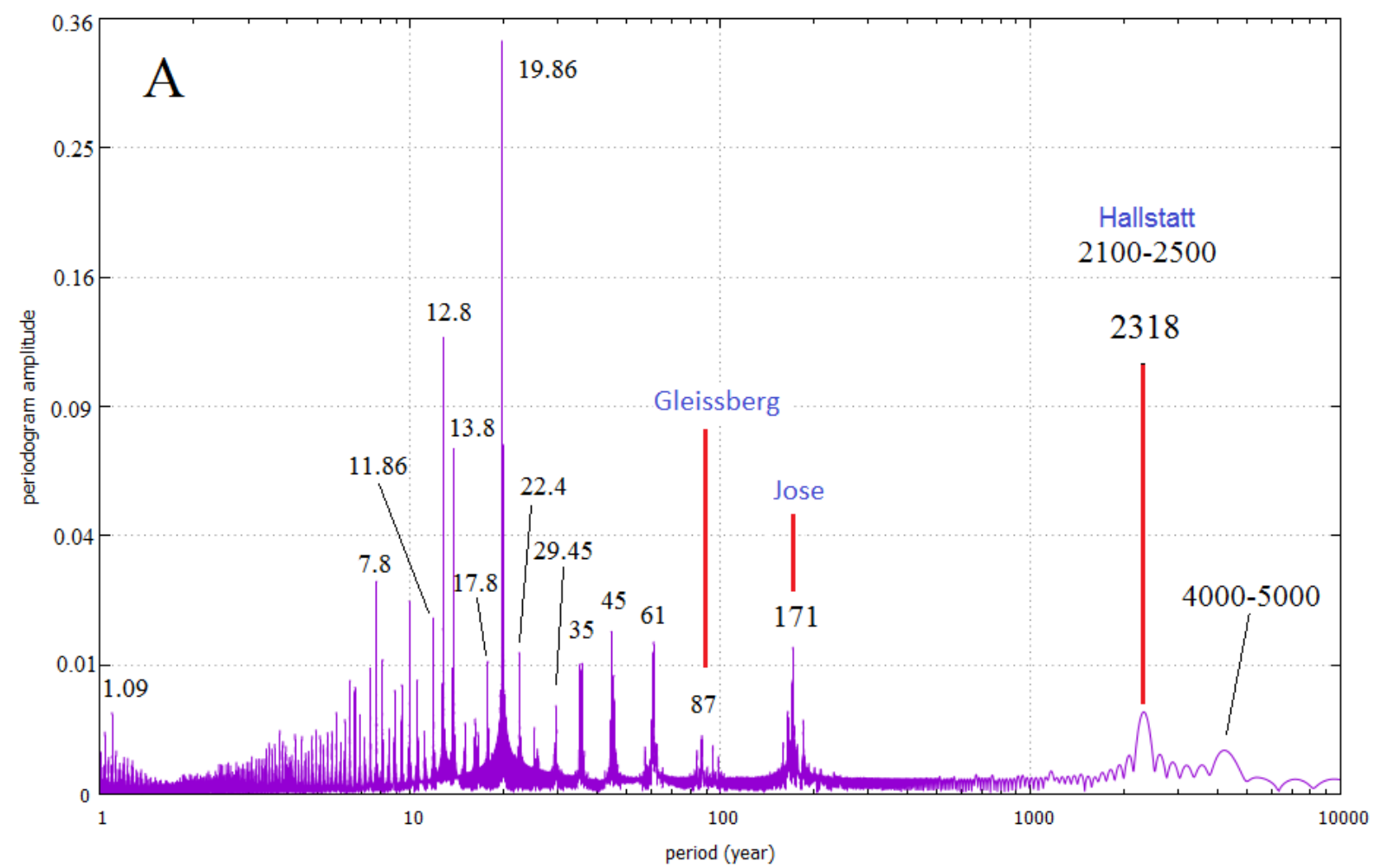

Stable orbital resonances

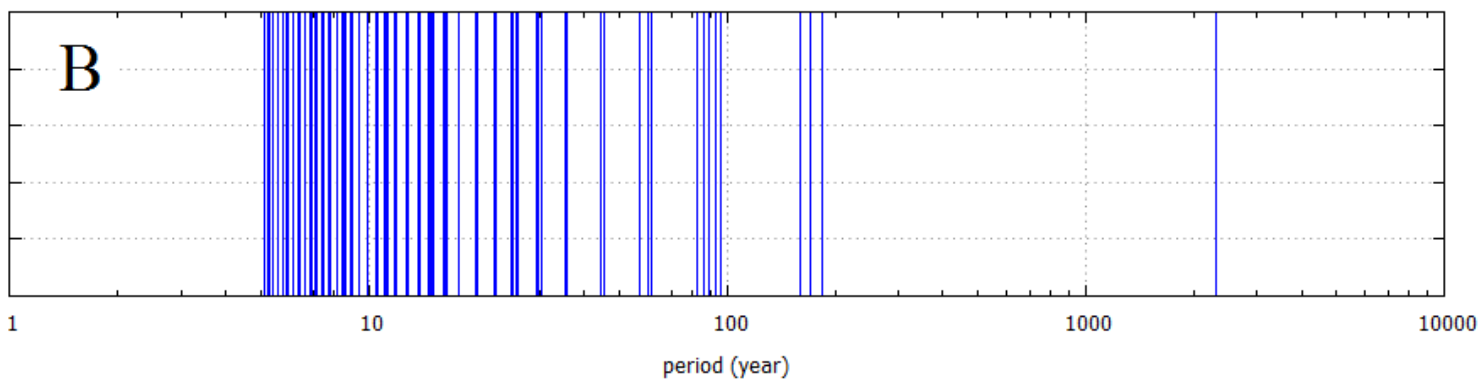

Figure 4: $[\mathrm{A}]$ Peridogram of the eccentricity record of the Sun-PMC orbit depicted in Figure 3. The spectral peak corresponding at the Hallstatt period at $2318 \mathrm{yr}$ is well visible. [B] The blue bars represent the stable planetary resonances of the solar system generated by Jupiter, Saturn, Uranus and Neptune for periods larger than 5 years: see also Table 1 . Note the accurate correspondence between these resonances and the spectral peaks depicted in $[\mathrm{A}]$.

more circular ones $(e \approx 0.590)$, that is while the system is bursting outward. Analogously, a smaller production of radionucleotide particles occurs while the Sun-PMC orbit evolves from statistically more circular shapes $(e \approx 0.590)$ to statistically more elliptical ones $(e \approx 0.598)$, that is while the system is bursting inward.

Figure 5 (lower panels) shows trajectories of the Sun-PMC orbits when these are statistically more elliptical (upper list of lower panels, the average eccentricity is $e \approx 0.598$ ) and when these are statistically more circular (lower list of lower panels, the average eccentricity is $e \approx 0.590$ ). The chosen time intervals were 344 year long that is twice the $172 \mathrm{yr}$ harmonic revealed in the periodogram of Figure 4. The upper list of these panels reveals that during these periods the Sun-PMC orbits are skewed with large regions that are rarely visited and the trajectory appears developing mostly within a 5 AU radius, which is the orbit of Jupiter, but sometimes it also clearly exceeds the 7 AU radius distance from the Sun (red curve). The 

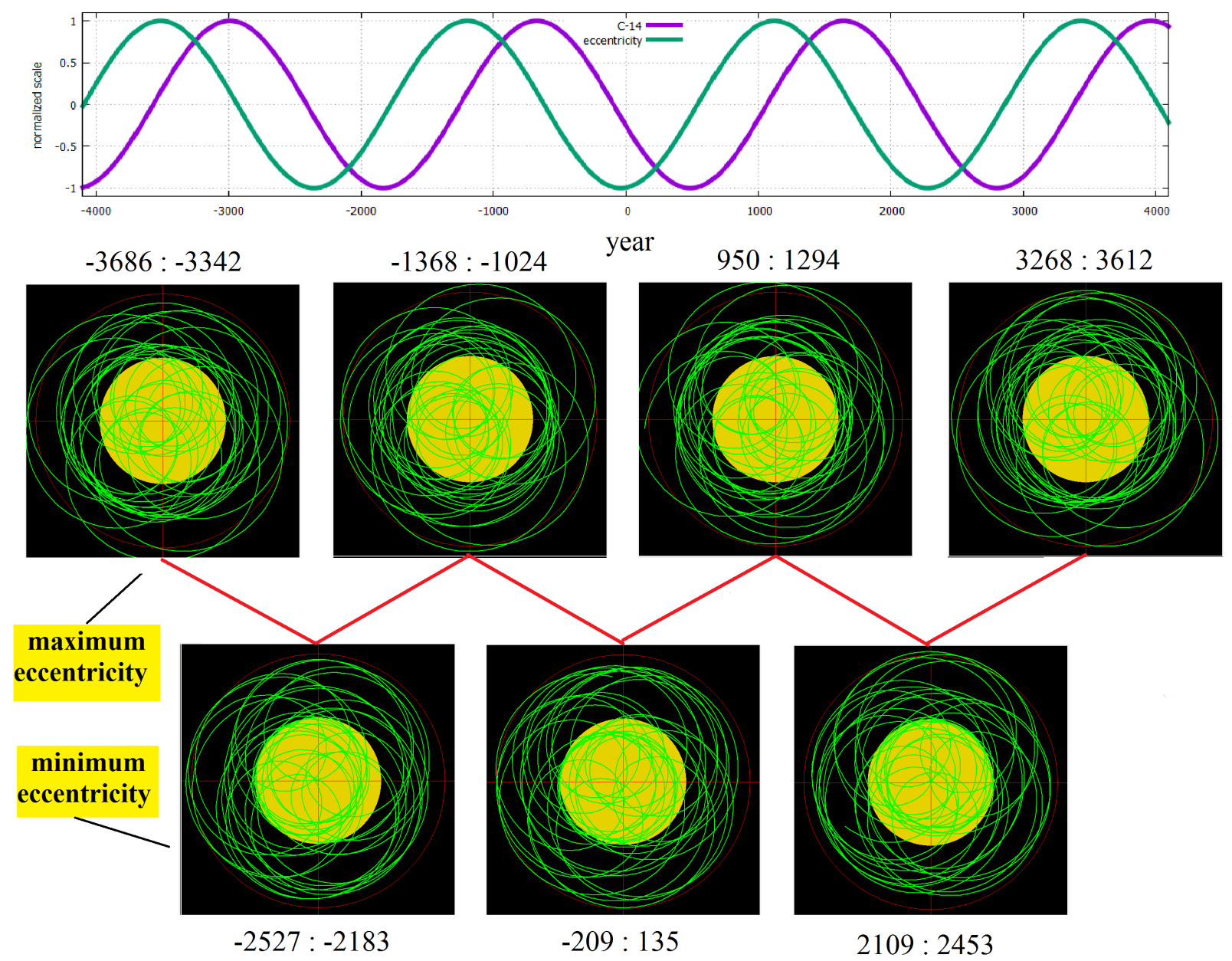

Figure 5: (Top) 2300-2400 yr harmonics referring to the $\Delta^{14} C(\%)$ record depicted in Figure 1 and the eccentricity record depicted in Figure 3. Note the $\pi / 2$ phase shift. (Bottom) Trajectory of the PMC relative to the Sun during intervals of 344 years referring to periods of the eccentricity maxima (above, more disordered, open orbits) and minima (below, more ordered, closed orbits). The yellow disk radius is about $3.5 \mathrm{AU}$, while the red circle radius is about $7 \mathrm{AU}$. The transition periods (red segments) from the eccentricity maxima to minima correspond to maxima in radionucleotide production, while the transition periods from the eccentricity minima to maxima correspond to minima in radionucleotide production.

lower list of panels reveals that during these periods the Sun-PMC orbits are more regular, more circular, symmetric and more uniformly cover all areas within a 7 AU radius distance from the Sun.

The dynamics observed in Figure 5 is also reminiscent at the larger Hallstatt time scale of the trefoil ordered and disordered state of the inertial motion of the Sun which is correlated to the gran maxima and minima of solar activity, respectively, as suggested by Charvátová (2000, 2009) inspired by the 178.7 yr cycle found by Jose (1965). However, as Figure 5 shows, here it is during the transition periods from an orbital state to the other that correlates with periods of maximum or minimum radionucleotide production.

\section{Analysis of the pericycle and apocycle orbital arcs}

The solar system pulses driven by the revolution of its planets around the Sun and the major harmonic period of this dynamics within the 200-10,000 yr time scale is $2318 \mathrm{yr}$ : this period perfectly corresponds to the 2100-2500 yr Hallstatt oscillation. However, in the chosen observable, the eccentricity variation of the 

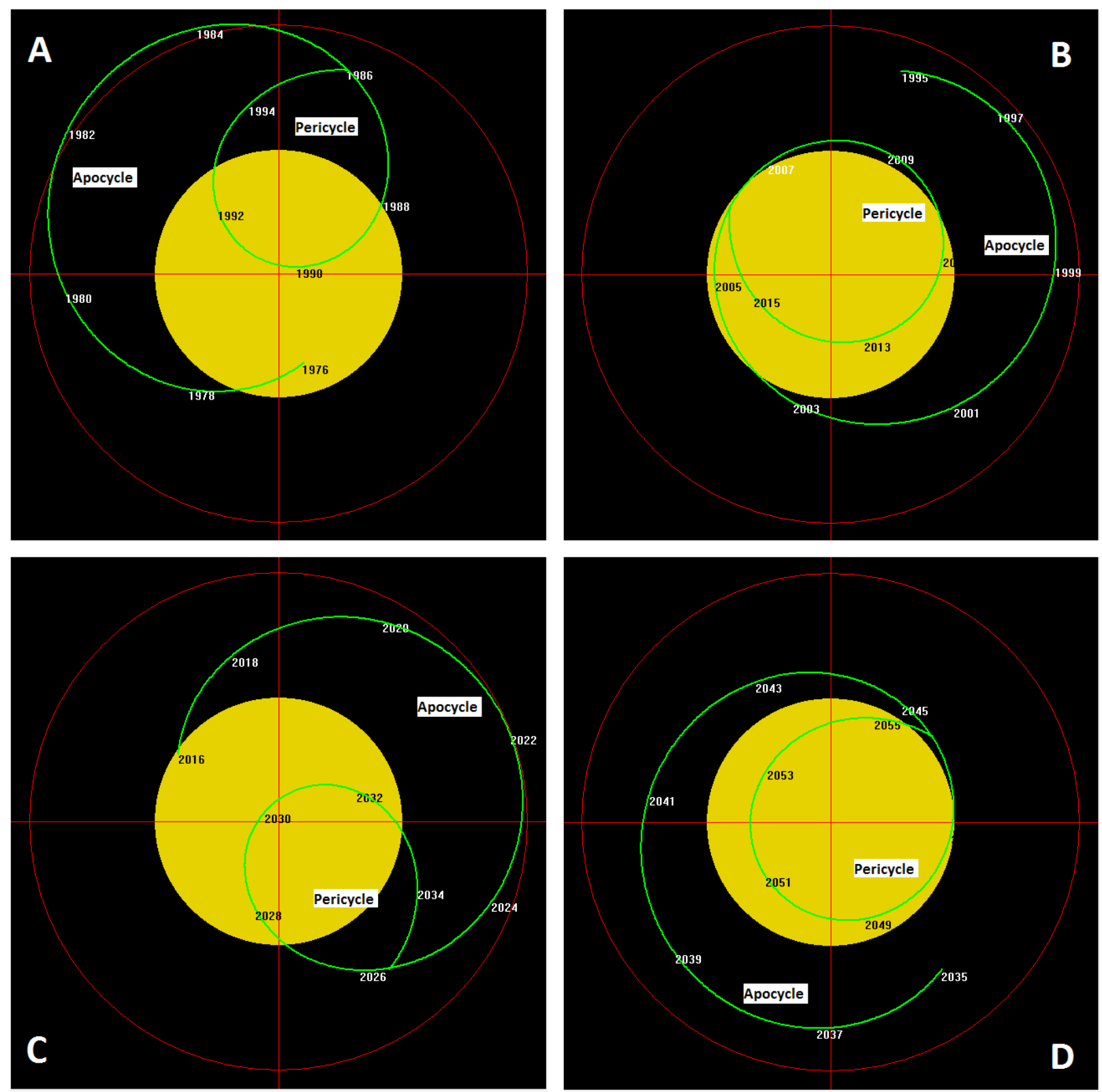

Figure 6: Motion of the PMC relative to the Sun from 10/6/1976 to $17 / 11 / 2055$. The diagrams depict four contiguous orbits made of one apocycle (external larger orbit) and one pericycle (internal smaller orbit). (Solar Orbit Simulator, http://arnholm.org/astro/sun/sc24/sim1/). The yellow disk radius is about 3.5 AU, while the red circle radius is about $7 \mathrm{AU}$.

Sun-PMC orbits, this slow oscillation is relatively small: to make it visible in Figure 3 we needed to plot it magnified by 10 .

It is important to search for a more specific astronomical origin for the Hallstatt-cycle that could stress the above dynamical characteristics of the Sun-PMC orbit. The search for a more appropriate orbital proxy is addressed in this section.

As evident in Figure 6, the Sun-PMC dynamics is characterized by a series of unit cycles made of an apocycle, or external large orbit, and a pericycle, or internal small orbit (cf. Piovan and Milani, 2006). During each apocycle the PMC moves in an arc in which it reaches a maximum speed and distance from the Sun. In the following pericycle the PMC enters into a helical coil in which it reaches a minimum speed 

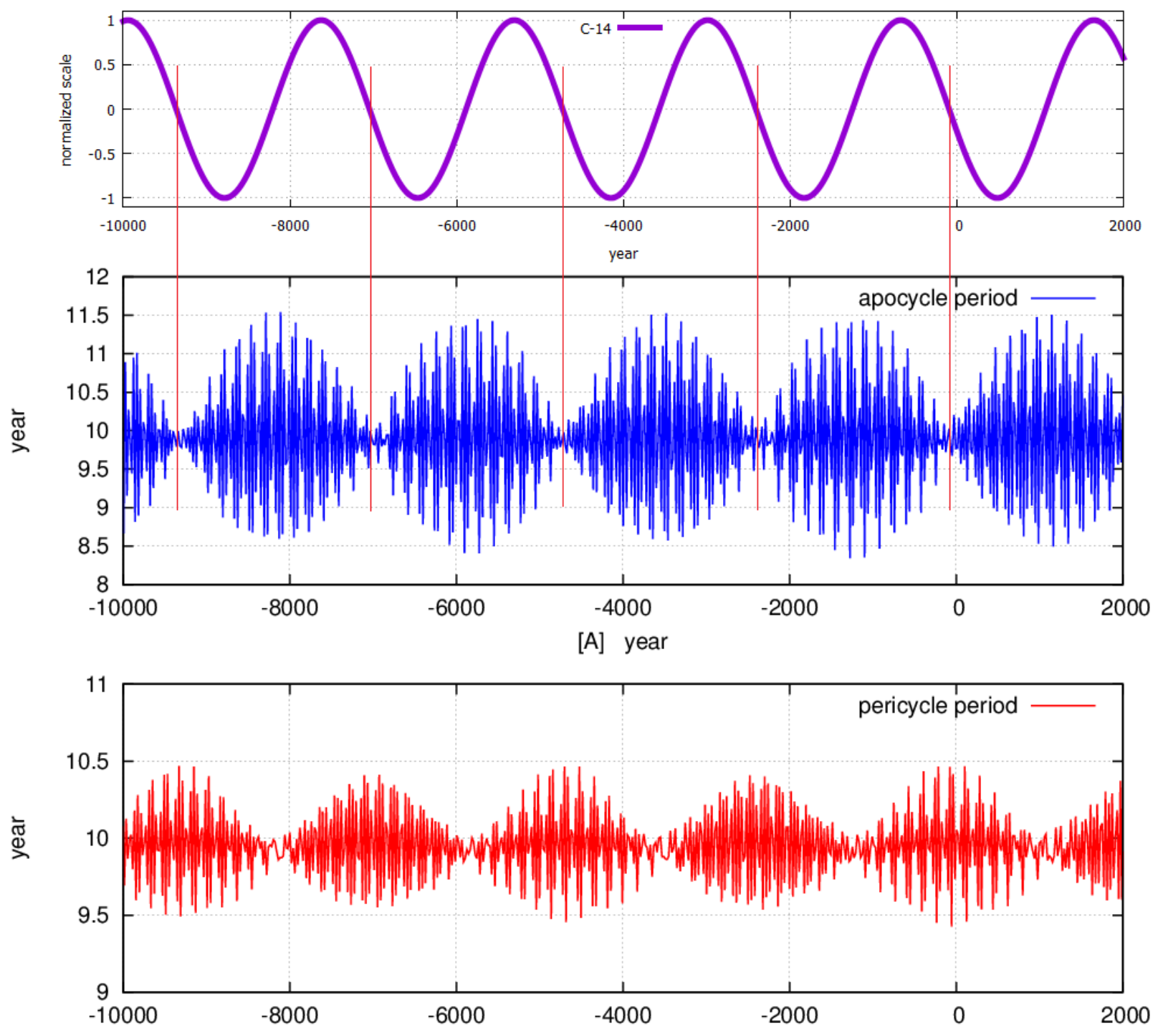

[B] year

Figure 7: Upper panel: the Hallstatt oscillation found in $\Delta^{14} C(\%)$ record, as depicted in Figure 1B. Lower panels show the time periods of the apocycles [A] and pericycles [B] of the PMC relative to the Sun. The upper panel Hallstatt oscillation is approximately in phase quadrature $(\phi=\pi / 2$ and $\phi=-\pi / 2)$ with the beat oscillation depicted in the lower panels as the red vertical lines show.

and distance from the Sun before returning to a position very near to the point where it has entered in the pericycle. This exit point is the beginning of the following apocycle. Figure 6 shows 4 consecutive full (apocycle plus pericycle) orbits from 10/6/1976 to 17/11/2055.

The orbit sections depicted in Figure 6 vary substantially in time. Sometimes the apocycles are very different from the pericycle (as in the figure). Other times they have similar amplitudes. This variation is due to the relative position of the various planets, in particular of the large Jovian ones. Let us investigate in details the dynamics of these apocycles and pericycles.

Figure 7A shows the time periods of the subsequent apocycles, $P_{a}$, while Figure 7B shows those of the following pericycles, $P_{p}$. The apocycle periods average about $\mu_{a}=9.91 \mathrm{yr}$ and vary from this mean up to a \pm 1.5 years while the pericycle periods average about $\mu_{p}=9.95 \mathrm{yr}$ and vary from their mean up to a \pm 0.5 years. The sum of the two average periods is 19.86 years that corresponds to the conjunction period between 

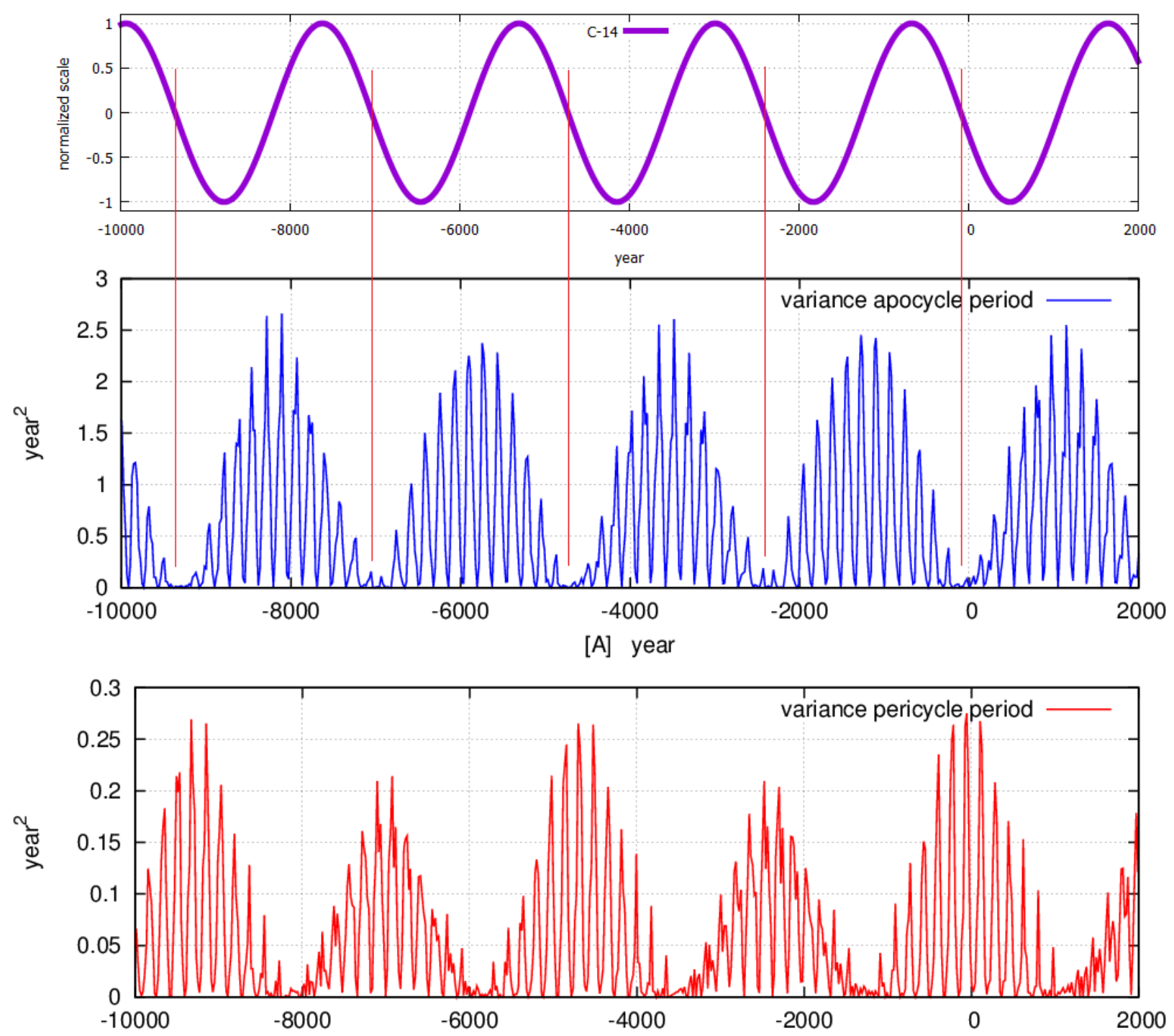

[B] year

Figure 8: Upper panel: the Hallstatt oscillation found in $\Delta^{14} C(\%)$ record, as depicted in Figure 1B. Lower panels: $[\mathrm{A}]$ and $[\mathrm{B}]$ are calculated from the records depicted in Figure $7 \mathrm{~A}$ and $\mathrm{B}$, respectively, as the square of their volatility from the mean. The upper panel Hallstatt oscillation is approximately in phase quadrature $(\phi=\pi / 2$ and $\phi=-\pi / 2)$ with the oscillation depicted in the lower panels as the red vertical lines show.

Jupiter and Saturn. The upper panel of Figure 7 shows an Hallstatt oscillation at 2318 yr period found in $\Delta^{14} C(\%)$ record, as depicted in Figure 1B, to show that its phase is about $\phi=\pi / 2$ and $\phi=-\pi / 2$ with the beat oscillation depicted in the lower A and B panels, respectively.

Figures $8 \mathrm{~A}$ and $8 \mathrm{~B}$ depict two records calculated from the periods depicted in Figure $7 \mathrm{~A}$ and $\mathrm{B}$, respectively, as the square of the volatility from their mean $\mu$, that is as: $\left(\Delta P_{a}\right)^{2}=\left(P_{a}-\mu_{a}\right)^{2}$ and $\left(\Delta P_{p}\right)^{2}=\left(P_{p}-\mu_{p}\right)^{2}$, respectively. This operation was chosen to make even more evident the 2100-2500 year oscillation present in these records. The upper panel of Figure 8 shows the Hallstatt oscillation found in $\Delta^{14} C(\%)$ record, as depicted in Figure $1 \mathrm{~B}$, to show that its phase is about $\phi=\pi / 2$ and $\phi=-\pi / 2$ with the oscillation depicted in the lower A and B panels, respectively.

Finally, Figure 9A shows, for each full apocycle plus pericycle unit, the times requested by the PMC to move from the minimum to the following maximum distance from the Sun. Figure 9B, instead, shows for each orbit the times requested by the PMC to move from the maximum to the following minimum distance 

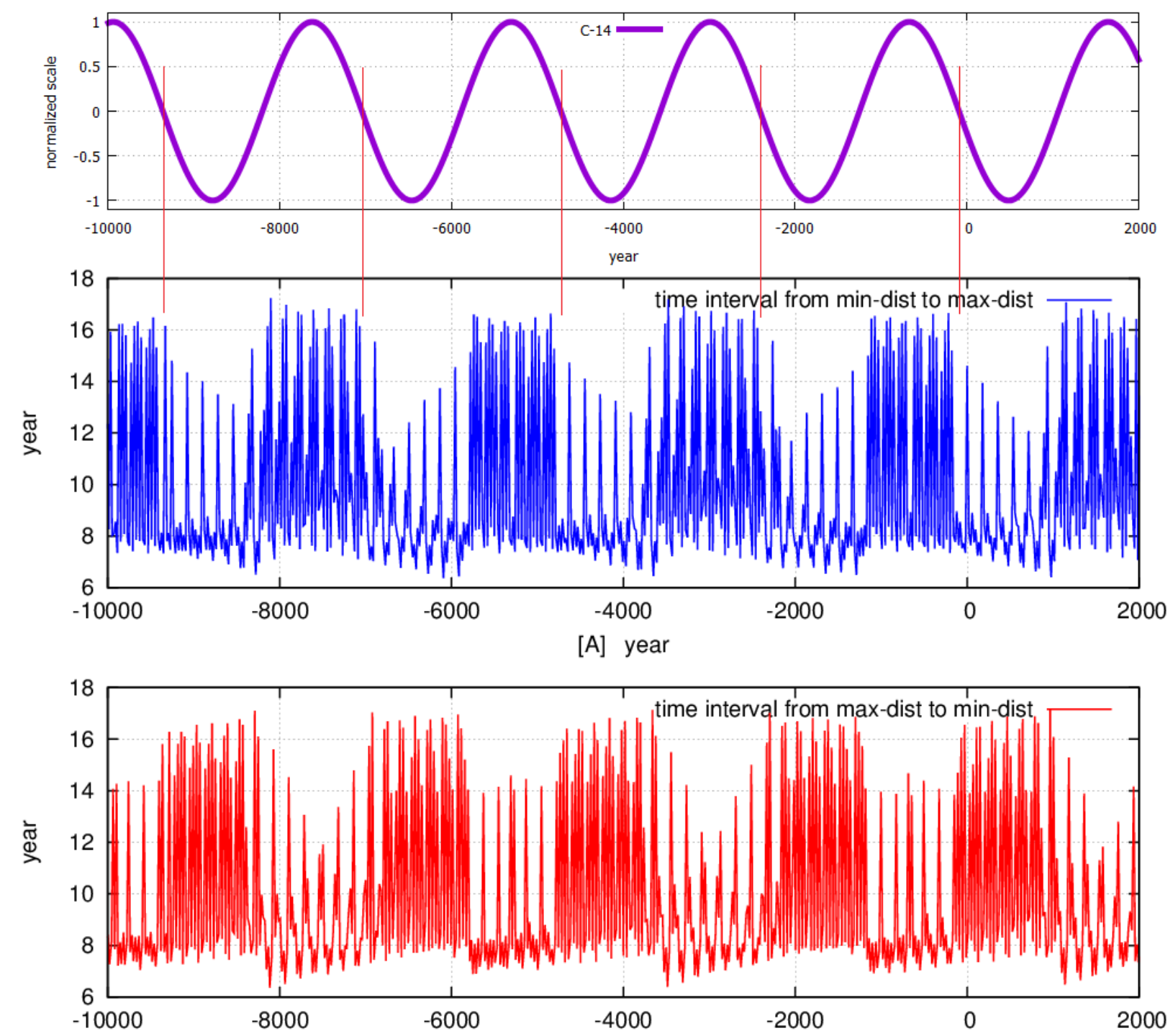

[B] year

Figure 9: Upper panel: the Hallstatt oscillation found in $\Delta^{14} C(\%)$ record, as depicted in Figure 1B. Lower panels: [A] Sequence of time intervals requested by the PMC to move from its minimum to its maximum distance from the Sun; [B] Sequence of time intervals requested by the PMC to move from its maximum to its minimum distance from the Sun. The upper panel Hallstatt oscillation is approximately in phase $(\phi=0$ and $\phi=-\pi)$ with the oscillation depicted in the lower panels as the red vertical lines show.

from the Sun. The upper panel of Figure 9 depicts the Hallstatt oscillation found in $\Delta^{14} C$ (\%o) record, as in Figure 1B, to show that its phase is about $\phi=0$ and $\phi=\pi$ with the oscillation depicted in the lower A and B panels, respectively.

The time sequences depicted in Figures 7-9 clearly put in evidence a strong oscillation of about 2318 years. Figure 7 reveals the presence of a major beat frequency with such a period, while Figures 8 and 9 reveal a direct 2318 yr oscillation. Moreover, the phase coincidence observed in Figure 9 between the Hallstatt oscillation found in $\Delta^{14} C(\%)$ record and in that observed in the astronomical record suggests that on the 2100-2500 yr time scale the cosmic ray flux reaching the Earth is higher, when during intervals of about 172 years, within the pericycle-apocycle orbits, the time required by the PMC to move from the minimum to the maximum distance from the Sun varies from about 8 to 16 years while the time required 


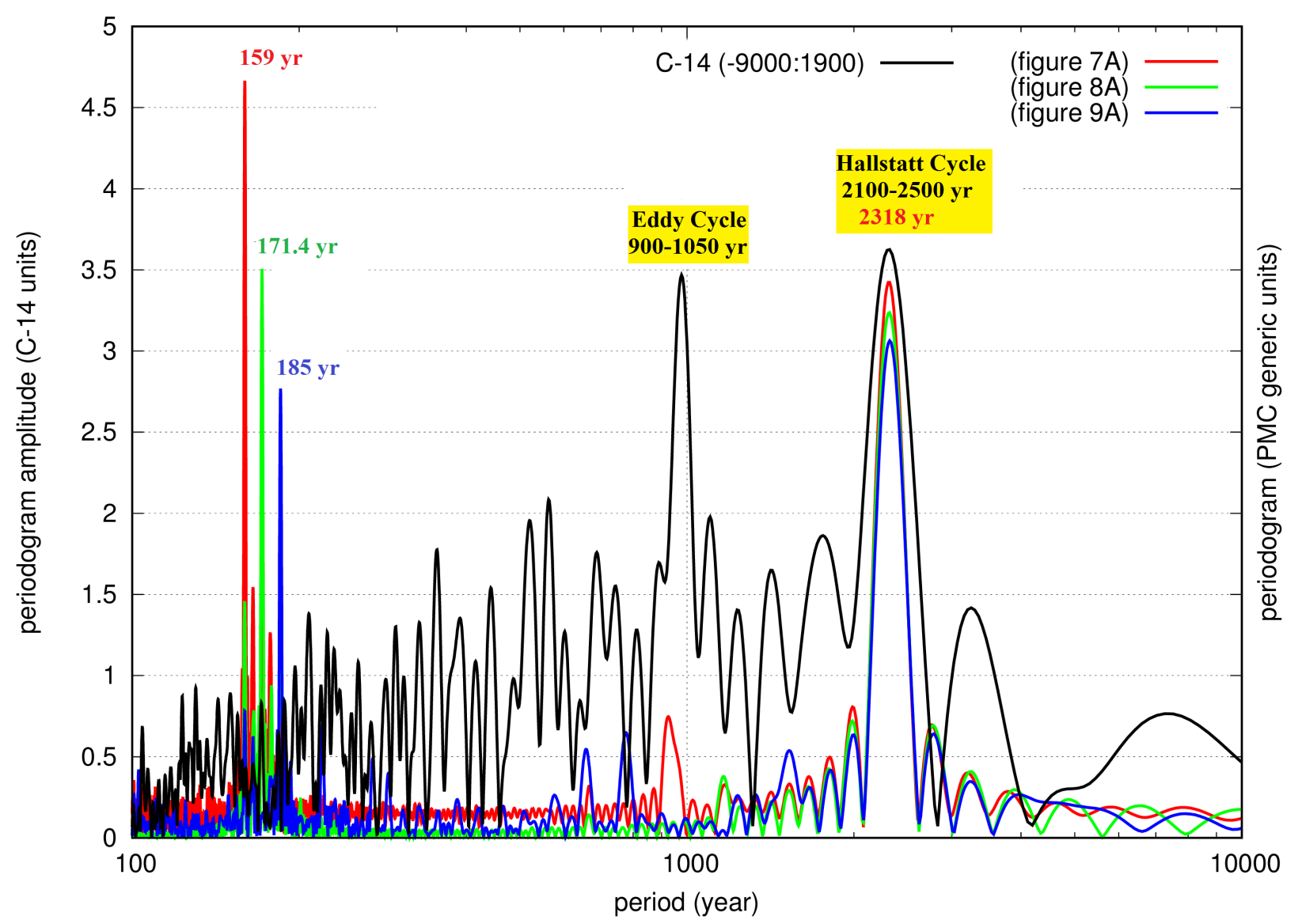

Figure 10: (Black curve) Periodogram of the $\Delta^{14} C$ record from -9000 B. C. to 1900 A. D. that is depicted in Figure 1B. (Colored curves) Same for the Sun-PMC orbital records depicted in Figures 7A, 8A and 9A spanning from 10,000 B. C. to 10,000 A. C.. Note the common spectral peaks at 2100-2500 yr period which are centered at the orbital resonance period of $2318 \mathrm{yr}$. The three peaks on the left are at about 159 years, 171.4 years and 185 years: the orbital resonances discussed in Section 3. The Eddy and Hallstatt spectral peaks have a $95 \%$ statistical confidence with respect to a red-noise background using the Multi Taper Method, MTM (Ghil et al., 2002).

by the PMC to move from the maximum to the minimum distance from the Sun varies from about 7 to 14 years; on the contrary, the minima of the radionucleotide production occurred, when the time required by the PMC to move from the minimum to the maximum distance from the Sun varies from about 7 to 14 years while the time required by the PMC to move from the maximum to the minimum distance from the Sun varies from about 8 to 16 years.

The power spectra functions depicted in Figure 10 show that the $\Delta^{14} C$ record depicted in Figure $1 \mathrm{~B}$ and the Sun-PMC orbital records depicted in Figures 7-9 share a very large common frequency peak at 2100-2500 year period centered at the stable orbital resonance of 2318 yr. These spectral peaks have a $95 \%$ statistical confidence against red-noise background (Ghil et al. 2002).

Figure 10 shows also that the radionucleotide record presents a significant 900-1050 year Eddy oscillation that has been extensively found in ${ }^{14} \mathrm{C},{ }^{10} \mathrm{Be}$ and climate records throughout the Holocene (Bond et al. 2001; Kerr, 2001) and has been modeled involving the orbits of Jupiter and Saturn and the 11-year solar cycle (Scafetta, 2012a, 2014a). The additional multi-secular minor spectral peaks present in the $\Delta^{14} C$ record are not further discussed here, but they have been also found among the planetary harmonics such as the following periods: 104, 130, 150, 171, 185, 208, 354, 500-580 yr (e.g.: Abreu et al., 2012, Scafetta, 2014a). 


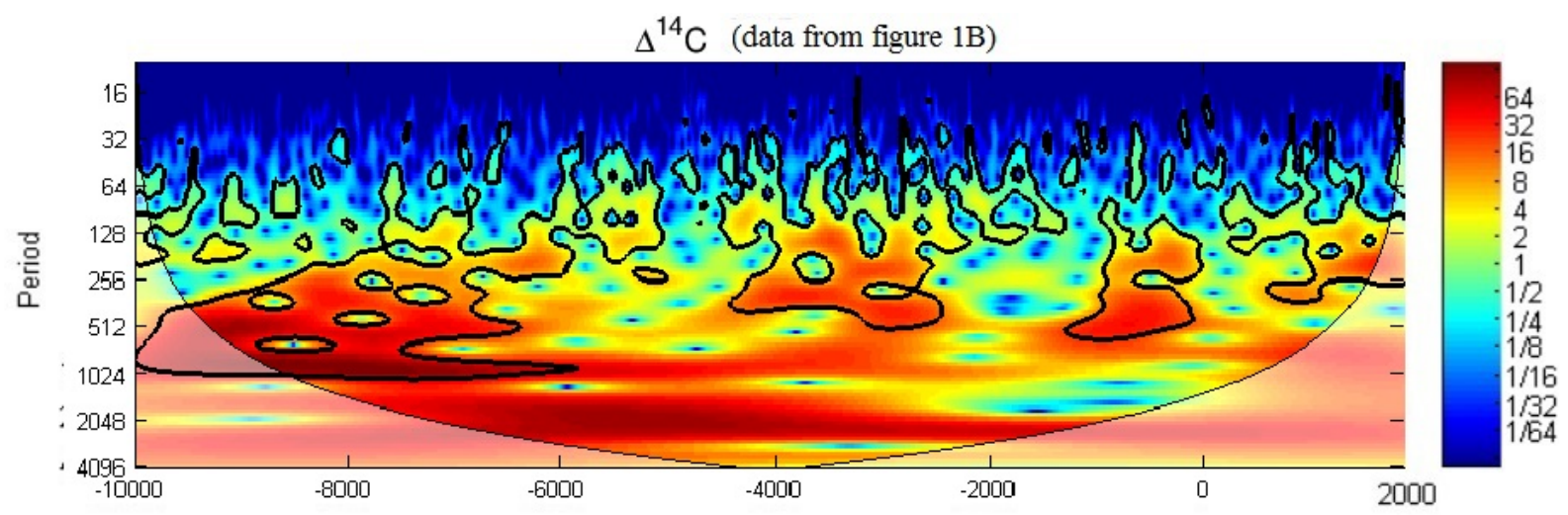

(data from figure $7 \mathrm{~A}$ )

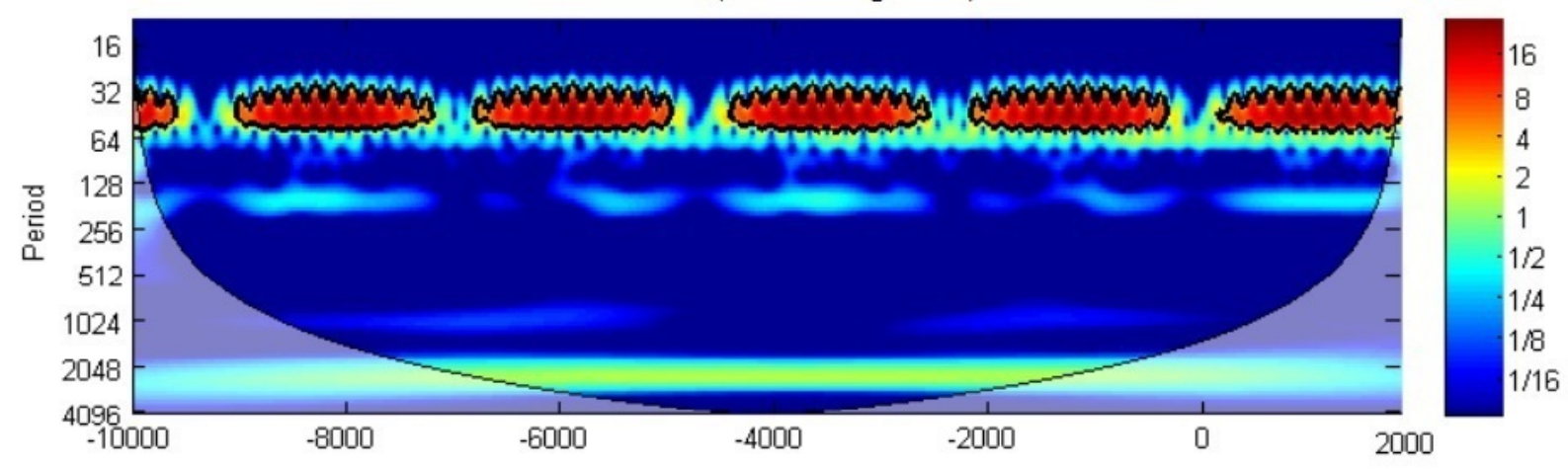

(data from figure $8 \mathrm{~A}$ )

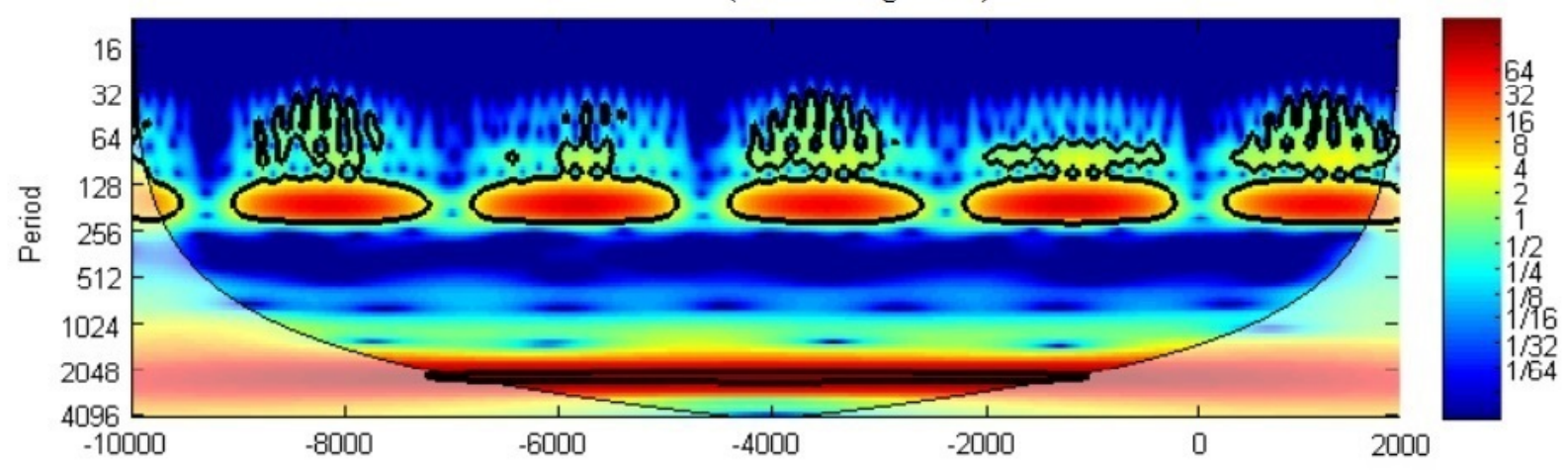

(data from figure 9A)

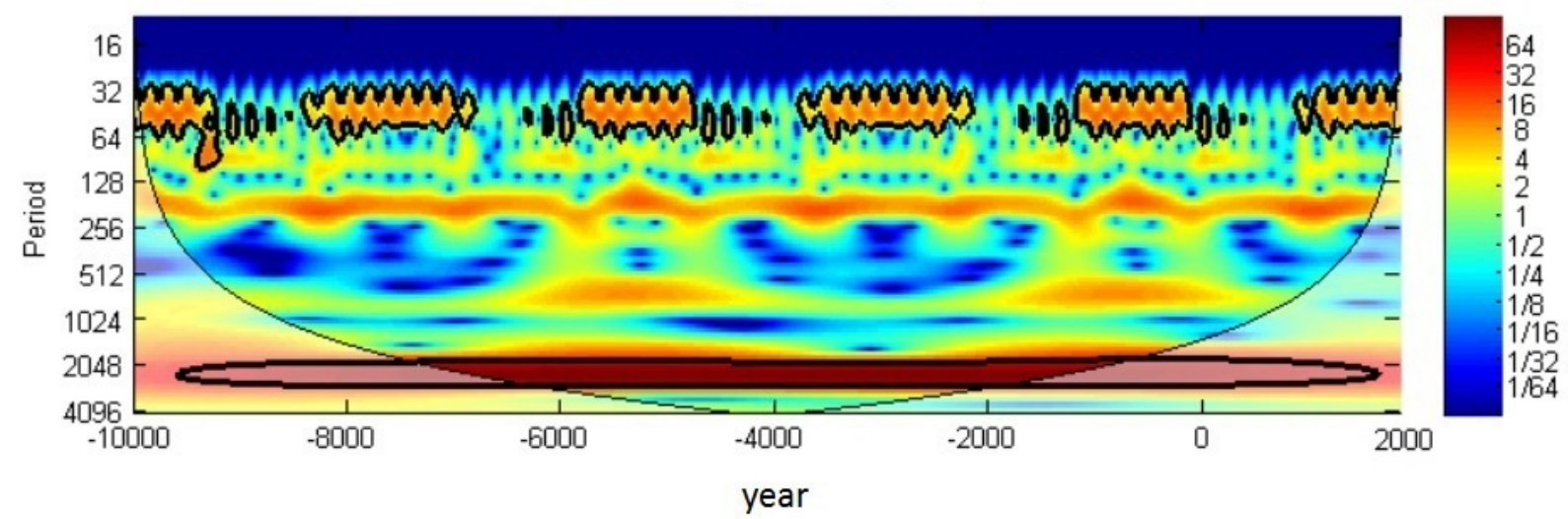

Figure 11: Continuous wavelet transforms of the records depicted in Figures 1B, 7A, 8A and 9A, respectively.

Figure 10 also reveals that the chosen orbital measures present spectral peaks at about 159 years (from Figure 7A), 171-172 years (from Figure 8A) and 185 years (from Figure 9A), which are also stable orbital 

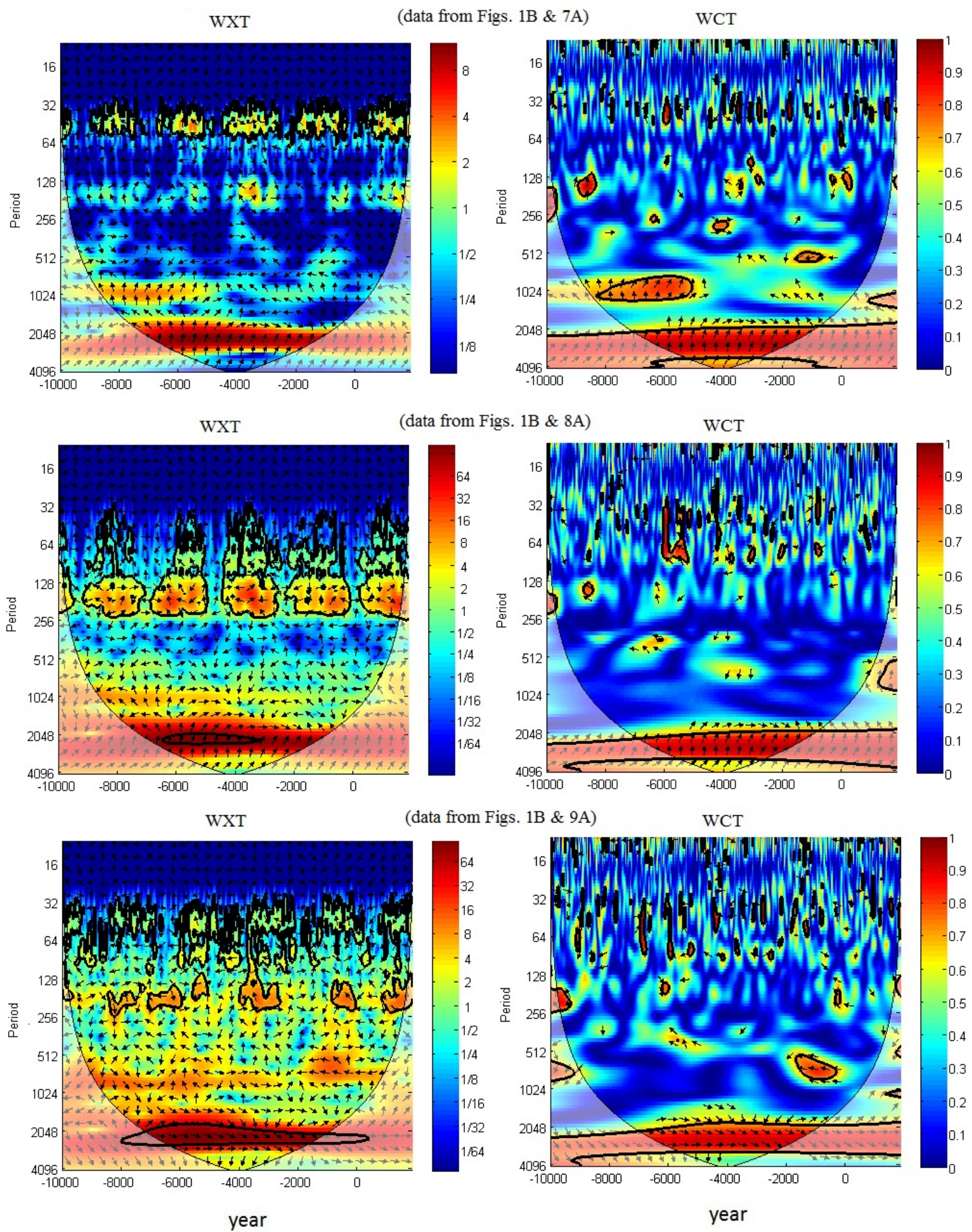

Figure 12: (Left panels) The cross wavelet transform (XWT) and (right panels) the wavelet coherence (WTC) between the $\Delta^{14} C$ record depicted in Figure $1 \mathrm{~B}$ and each of the Sun-PMC motion records depicted in Figures $7 \mathrm{~A}, 8 \mathrm{~A}$ and $9 \mathrm{~A}$, respectively. The red areas surrounded by the black line satisfy the $95 \%$ confidence level.

resonances as discussed in Section 3. The 171-172 and 185 yr periods are visible in the $\Delta^{14} C$ record although very small, but they appear well in other solar records (cf: McCracken et al., 2014, Sharp, 2013). 
Figure 11 shows the continuous wavelet transforms of the records depicted in Figures 1B, 7A, 8A and 9A, respectively. Also these four panels show that the four records share a significant harmonic at about 2100-2500 yr period.

Finally, we study the spectral coherence between the $\Delta^{14} C$ record and the chosen astronomical records. Figure 12 shows in the left panels the cross wavelet transform (XWT) and in the right panels the wavelet coherence (WTC) between the $\Delta^{14} C$ record depicted in Figure $1 \mathrm{~B}$ and each of the Sun-PMC motion records depicted in Figures 7A, 8A and 9A, respectively (Grinsted et al., 2004). The cross wavelet transform finds regions in time frequency space where the time series show high common power. The wavelet coherence finds regions in time frequency space where the two time series co-vary but does not necessarily have high power.

As clearly shown in Figure 12, the six panels demonstrate that the $\Delta^{14} C$ record and the chosen astronomical records share a coherent frequency at about $2100-2500$ year period with a $95 \%$ statistical confidence against red noise background.

\section{Discussion and Conclusion}

Several experimental evidences demonstrate that throughout the Holocene the ${ }^{14} C$ atmosphere concentration has varied cyclically in time (e.g. Damon and Linick, 1986, Houtermans, 1971, Kromer et al., 1998, and many others). An observed large oscillation has a period of about 2100-2500 years. This oscillation is known in the scientific literature as the Hallstatt cycle. As discussed in the Introduction, the presence of a fundamental harmonic at such a period has been confirmed in numerous studies and found also in ${ }^{10} B e$ and climate records. For example, recently McCracken et al. (2013) confirmed an oscillation with period centered between 2300 and 2320 using Fourier amplitude spectrum for GRIP ${ }^{10} \mathrm{Be}$, the modelled estimate of the ${ }^{14} \mathrm{C}$ production rate and the modulation function (in $\mathrm{MeV}$ ) computed from the EDML and GRIP ${ }^{10} B e$ data, and the INTCAL09 ${ }^{14} \mathrm{C}$ record.

A fundamental scientific issue is to understand the origin of such an oscillation. It is legitimate to claim that it is an internal climate or solar oscillation, but in absence of an explicit physical mechanism this interpretation remains an unproven hypothesis. This leaves open the possibility for an external astronomical origin of the observed oscillation. It is observed that the only well-known harmonic generator of the solar system is provided by the gravitational and electromagnetic oscillations induced by the revolution of the planets around the Sun.

Thus, we have hypothesized that the Hallstatt oscillation found in radionucleotide and climatic records could be the result of a specific orbital resonance within the solar system. A search of the stable resonances involving the four outer giant planets - Jupiter, Saturn, Uranus and Neptune - has determined that, indeed, there exists a major stable resonance with a period of 2318 years. This stable resonance is also the only one for period larger than 200 years among those listed in Table 3. Since this resonance is perfectly coherent to the Hallstatt oscillation found in radionucleotide and climate records, this is unlikely a coincidence: we can name this resonance as the Hallstatt H-resonance of the solar system.

We have also theoretically determined a large number of additional stable orbital resonances of the solar system and many of their periods (e.g. about 20 yr, 44-46 yr, 57-62 yr, 82-97 yr, 159-171-185 yr) are also typically found in solar, aurora and climate records throughout the Holocene (e.g.: Ogurtsov et al., 2002, McCracken et al., 2014, Sharp, 2013, Scafetta and Willson, 2013a, Scafetta, 2014a; Vaquero et al., 2002, and many others).

Inspired by the Milanković (1930)'s theory linking the variation of the Earth's orbit eccentricity to the glacial cycles, we test whether the Hallstatt cycle could derive from, and therefore be revealed by, the overall variation of the circularity of the solar system disk that could eventually modulate the solar wind intensity 

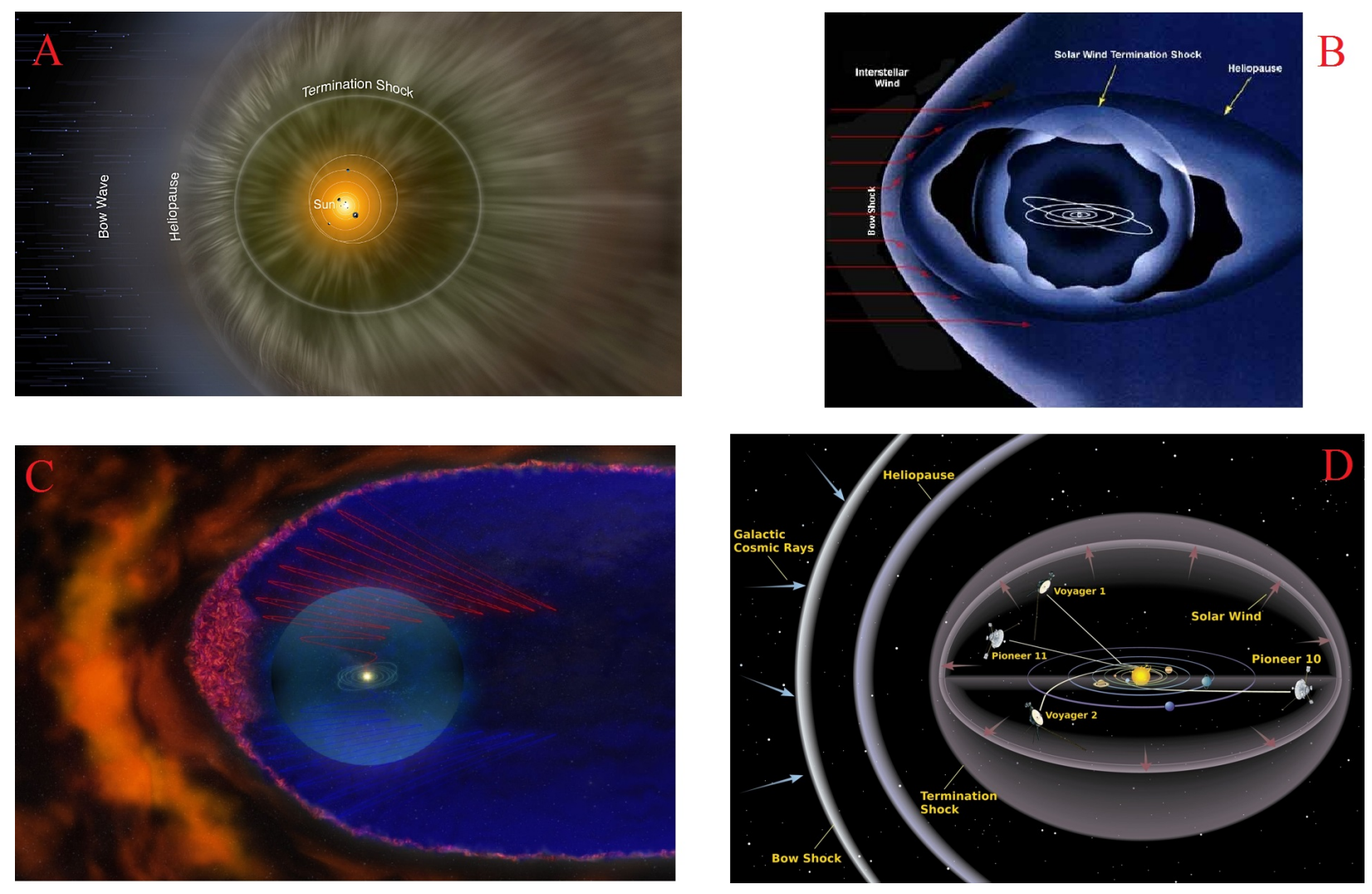

Figure 13: Artist representations of the heliosphere of the solar system with highlighted the solar wind termination shock, the heliopause, the bow shock and the incoming interstellar wind, that is the cosmic ray flux which is mostly deflected at the heliopause. These illustrations shows how the sun's activity pushes out cosmic radiation from outside of the solar system. These and other artist representations of the heliosphere have been published by NASA (credit to Howell, 2014, Phillips, 2011, and others).

and direction and therefore also the incoming cosmic ray flux and the interplanetary dust concentration around the Earth. We chose to study the orbit of the planetary mass center (PMC) relative to the Sun and used the instantaneous eccentricity vector function (e.g. Mungan, 2005) applied to the Sun-PMC orbit to determine the eccentricity variation of this virtual planet from 13,000 B. C. to 17,000 A. D. Using spectral analysis we have demonstrated that this observable presents a significant oscillation with a 2318 yr period together with a number of already known oscillations associated to the orbital periods of the planets at scale shorter than 200 years. Figure 4 stresses that the 2318 yr period peak is the most relevant in the spectral range between 200 and 10,000 yr indicating that this oscillation dominates this time scale range. Thus, there exists a rhythmic contraction and expansion pattern of the solar system induced by the planets; this pulse is spectrally coherent to the Hallstatt oscillation found in nucleotides and climate records.

In particular, we found a $\pi / 2$ phase shift between the $2100-2500 \mathrm{yr}$ curves present in the variation of ${ }^{14} C$ record and the solar system eccentricity function. Thus, on the Hallstatt-cycle time scale a larger production of radionucleotide particles, i.e. the occurrence of a stronger cosmic rays flux toward the inner region of the solar system, occurs while the Sun-PMC orbit evolves from statistically more elliptical shapes $(e \approx 0.598)$ to statistically more circular ones $(e \approx 0.590)$ : that is while the system is slowly imploding or bursting inward. Analogously, a smaller production of radionucleotide particles, i.e. the occurrence of a weaker cosmic ray flux toward the inner region of the solar system, occurs while the Sun-PMC orbit evolves from statistically more circular shapes $(e \approx 0.590)$ to statistically more elliptical ones $(e \approx 0.598)$ : that is while the system is slowly exploding or bursting outward. 


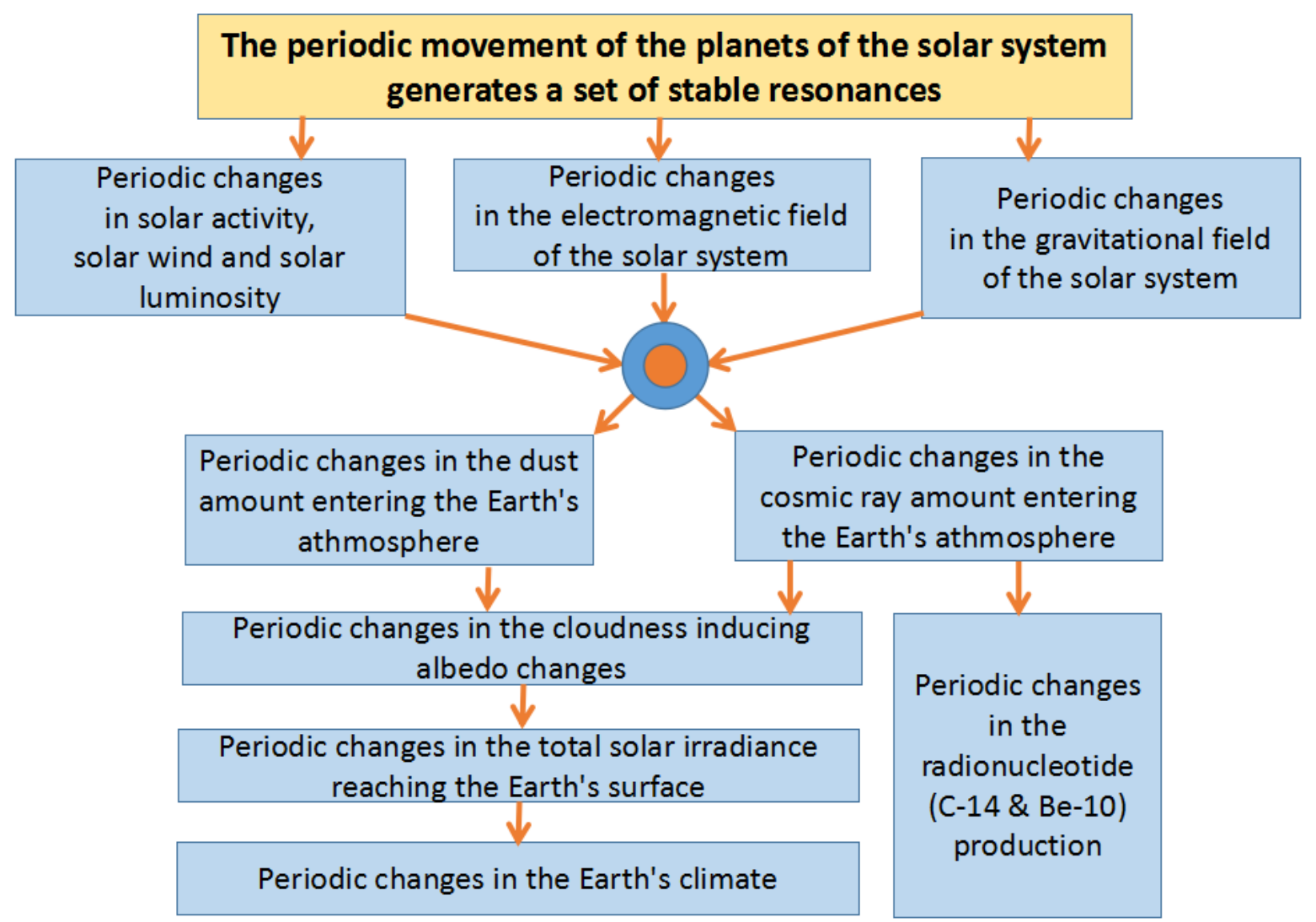

Figure 14: Schematic flow chart explaining a chain of mechanisms linking the periodic movement of the planets around the Sun with periodic changes in solar activity and climate change.

Finally, to better identify an astronomical proxy able to greatly stress the 2318-yr H-resonance, we analyzed how the pericycles and apocycles of the Sun-PMC orbits evolve (Piovan and Milani, 2006). We found that the time series of the periods of these orbits are characterized by a very prominent $2318 \mathrm{yr}$ oscillation that is perfectly coherent with the Hallstatt oscillation found in the investigated $\Delta^{14} C$ record with a statistical confidence above $95 \%$. These orbital proxies are also characterized by prominent 159, 171-172, and 185 yr oscillations, which correspond to other stable resonances of the solar system. Major harmonics within this spectral range, which was first identified by Jose (1965), is found in long solar activity proxy records (Solanki et al. 2004; Steinhilber et al., 2009) and in long historical aurora records (Scafetta and Willson, 2013a). The coherence at this time scale between our model and the data is revealed in Figure 12 by the WXT methodology. Other periodicities found in the eccentricity vector function of the Sun-PMC orbit, such as about the 20,30,45, 60, $87 \mathrm{yr}$ periods, are typically found among solar (cf. Ogurtsov et al. 2002), aurora and climate indexes (cf: Scafetta, 2010, 2012c, 2013; Scafetta and Willson, 2013a).

We found (e.g. Figure 9) that at the Hallstatt cycle maxima of the radionucleotide production occurred when the time required by the PMC to move from the minimum to the maximum distance from the Sun varies from about 8 to 16 years, while the time required to move from the maximum to the minimum distance varies from about 7 to 14 years throughout the full pericycle-apocycle pattern. On the contrary, the minima of the radionucleotide production occurred when the time required by the PMC to move from the minimum to maximum distance from the Sun varies from about 7 to 14 years while the time required by the PMC to move from the maximum to the minimum distance from the Sun varies from about 8 to 16 years. This suggests that, at this time scale, the cosmic ray flux increases (decreases) during periods of slower (faster) 
expansion and faster (slower) contraction of the Sun-PMC orbit wobbling. Thus, a faster expansion of the solar system prevents cosmic rays to enter in its inner regions, while a faster contraction favors a larger incoming of cosmic rays. This suggests a rhythmic modulation of the geometry of the heliopausa and/or of solar wind termination shock and, therefore, of the heliospheric magnetic field (Owens and Forsyth, 2013), which in the former case should become larger while in the latter should become smaller, inducing a larger or smaller deviation of the incoming cosmic rays, respectively.

Figure 13 shows a graphical representation of the heliosphere of the solar system with highlighted the solar wind termination shock, the heliopause, the bow shock and the incoming interstellar wind. A reader should note that the heliosphere is not stationary relative to the incoming cosmic rays, but it is wobbling like the Sun-PMC orbits that we have studied in this work. Since the planetary system is within the heliosphere this means that the interaction of the planets with the inner heliosphere can be relevant. In particular, magnetic field reconnections can capture and redirect solar wind shaping the heliosphere, which modulates the cosmic ray flux. The imploding-exploding dynamics revealed in our record could easily modulate the solar wind termination shock surface and, therefore, modulate the incoming cosmic ray flux. Potgieter (2013) summarizes mechanisms of solar modulation of cosmic rays in the heliosphere.

A related but complementary mechanism is that the displacements of the planets could directly or indirectly modulate the amount of interplanetary/cosmic dust falling on Earth. The cosmic-planetary dust is concentrated within the disk of the solar system where the Sun-PMC orbit evolves, including the region surrounding the Earth-Moon system, and regulates the intensity of the zodiacal light (cf.: Ermakov et al., 2009a b; Ollila, 2015). In fact, every day from 400 to 10,000 tons of dust enters in the Earth's atmosphere. The sizes of these particles vary from $0.001 \mu \mathrm{m}$ to several hundreds of micrometres and they are mostly made of common elements such as $\mathrm{Fe}, \mathrm{Mg}, \mathrm{S}, \mathrm{Al}, \mathrm{Ca}$, and $\mathrm{Na}$. Because these particles are very likely also charged by solar wind, they should be subject not only to gravitational forces but also to magnetic fields and to the solar wind itself (Divari, 1966). Therefore, interplanetary/cosmic dust can be driven by the planets (Divari, 1966). Once that these charged particles enter the Earth's atmosphere, they behave as efficient condensation nuclei of the atmospheric water vapor, which is a polarized molecule, in particular $\mathrm{Mg}, \mathrm{S}$, and $\mathrm{Na}$, and also help in forming clouds (cf.: Ermakov et al., 2009a b Ollila, 2015). The Sun-PMC wobbling could more easily disperse this dust away from the inner region of the solar system when its orbit expands fast and contracts slower as depicted in Figure 9 inducing the formation of less clouds on the Earth, and vice versa. Indeed, while long records of interplanetary/cosmic dust falling on Earth are not available to test this hypothesis, a record of historically recorded meteorite falls in China from 619 to 1943 A. D. has revealed harmonics such as 10.5, 14-15, 30 and 60-63 year oscillations (Scafetta, 2012c; Yu et al., 1983), which are also found among the main harmonics of the eccentricity function of the Sun-PMC orbit and among the stable resonances of the solar system (see Table 1). A modulation of the interplanetary/cosmic dust density surrounding the Earth-Moon system driven by the solar wind and the planets' magnetospheres may also contribute to explain why the Hallstatt cycle, and a number of other oscillations revealed in Figure 4, are also observed in climate records (e.g.: Levina and Orlova, 1993, O'Brien et al., 1995). Further research will better clarify the specific physical mechanisms involved in these processes.

In conclusion, our results clearly suggest that the velocity of the displacements of the Jovian planets (Jupiter, Saturn, Uranus and Neptune), which mainly determine the inertial motion of the Sun on the long time scales, also influences the solar activity and the intensity of its solar wind, and/or modifies the structure of the heliosphere. The latter would then modulate the incoming cosmic ray flux that produces radiocarbon and/or interplanetary/cosmic dust concentration and, simultaneously, they would regulate the Earth's climate by modulating the cloud system: see the schematic flow chart depicted in Figure 14. A possible chain of the involved mechanisms has been suggested by some authors (Kirby, 2007; Ollila, 2015. Scafetta, 2012c, 2013; Shaviv et al., 2014, Svensmark et al., 2009, Svensmark, 1998; Tinsley, 2008). Moreover, 
the fact that a specific set of planetary resonances may be modulating eliospheric, solar and climatic records may suggest why these records appear linked at multiple time scales, and even at the short ones (e.g.: Scafetta et al., 2004, Scafetta and West, 2006; Scafetta, 2009).

Although it is still uncertain how the planets could influence solar activity and/or the cosmic ray flux and/or the dust reaching the Earth, a planetary origin of solar and climate oscillations, which has been proposed since antiquity, has recently received a renewed attention in the scientific literature. Planetary theories of climate variations were widespread in ancient times and, in more recent times, a planetary theory of solar variation was proposed by Wolf (1859) to explain the 11-year solar cycle. Wolf hypothesized that the just discovered 11-year solar cycle could emerge from a combined influence of Venus, Earth, Jupiter and Saturn, which has been recently confirmed (Hung, 2007; Scafetta, 2012a b, Wilson, 2013). Stefani et al. (2016) speculated that the tidal oscillation of 11.07 years induced by the Venus-Earth-Jupiter system may lead to a 1:1 resonant excitation of the oscillation of the $\alpha$-effect. In general, a planetary origin of solar and climate oscillations is based on numerous empirical evidences at multiple time scales and some preliminary physical explanations (e.g.: Abreu et al., 2012, Charvátová, 2009, Cionco and Soon, 2014, Hung, 2007; Jakubcová and Pick, 1986; Jose, 1965; McCracken et al., 2013, 2014, Mörner, 2013, 2015; Puetz et al. 2014; Salvador, 2013, Scafetta, 2010, 2012a b, 2013, 2014a, 2016, Scafetta and Willson, 2013a b; Sharp, 2013; Solheim, 2013: Tan and Cheng, 2013: Tattersall, 2013a, Wilson, 2013).

Since the 19th century, the planetary theory of solar and climate variations has also received a number of critiques (some of the most recent critical studies include: Callebaut et al., 2012, Cameron, R. H., Schüssler, 2013, Cauquoin et al., 2014, Holm, 2014, 2015, Poluianovand Usoskin, 2014, Smythe and Eddy, 1977). However, several rebuttals of the critiques have also been published. The rebuttals can be summarized as follows: i) the Sun can react to a planetary tidal forcing because it is a nuclear fusion generator that might greatly amplify the modest gravitational tidal effect (e.g.: Scafetta, 2012b, Wolff and Patrone, 2010); ii) an additional electromagnetic coupling could link the Sun to the planets throughout the solar wind (e.g.: Scafetta and Willson, 2013b); iii) the solar-climate physics occurs throughout some heliosphere dynamics (e.g.: Scafetta and Willson, 2013b a); iv) the coupling between some astronomical and the solar-climate harmonics is very good when the appropriate astronomical proxies that takes into account multiple planets are constructed (e.g.: Scafetta, 2014a, 2016, Sharp, 2013, Wilson, 2013); v) the spectral coherence at the given harmonics is statistically significant above $95 \%$ when the calculations are done correctly and once the limits of the used analysis algorithms are properly considered (see also: Scafetta, 2014a, 2016); Monte Carlo techniques used to test the likelihood that multiple frequencies in solar records match planetary records show unambiguously that this probability is lower than $10^{-4}$ (e.g.: Abreu et al., 2014; Scafetta and Willson, 2013 b).

We also noted that once challenged, some critical authors responded by contradicting their previous claims. For example, Callebaut et al. (2012) argued against a planetary origin of the solar oscillation by also claiming that the five major identified solar periodicities - Schwabe $(\sim 11 \mathrm{yr})$, Hale $(\sim 22 \mathrm{yr})$, Gleissberg ( $\sim 88$ yr), Suess ( 203-208 yr) and Hallstatt ( 2300-2400 yr) cycles - were never successfully reproduced by papers advocating planetary influences on solar variability. However, when their claim was challenged (e.g.: Scafetta et al., 2013), Callebaut et al. (2013) acknowledged that "it is well-known that there are some periodicities that are common to solar activity and planetary motions" which make his further critique very weak as explained in Scafetta et al. (2013). Similarly, Holm (2014) argued that using windowed periodograms no spectral coherence between temperature records and the speed of the solar center of mass could be found at given frequencies such as at $20 \mathrm{yr}$ and $60 \mathrm{yr}$ periods. However, after that Scafetta (2014a) demonstrated that Holm used improperly the windowed periodogram, that is he used it with too short window segments to detect the signals, Holm (2015) acknowledged that "it is not hard to produce high coherence estimates at periods around 15-22 and 50-60 years between these data sets." Rebuttals of Holm (2015)'s further critiques 
were presented in Scafetta (2016).

Although several issues remain open to further investigations, the published scientific literature provides several evidences that solar and climate records are characterized by periodicities that are common to planetary motions at multiple time scales from a few months to several millennia. Moreover, no alternative explanations of these oscillations have been proposed by the critics. In other words, an alternative theory explaining the observed oscillations simply does not exist. The methodologies and results of the present paper contribute to this discussion showing compelling evidences that also the long Hallstatt (2100-2500 yr) oscillation likely has an astronomical origin linked to the internal dynamics of the solar system and its stable resonances such as those produced by Jupiter, Saturn, Uranus and Neptune at periods of $159 \mathrm{yr}, 171 \mathrm{yr}, 185$ yr and 2318 yr. From Figure 5, the next Hallstatt minimum in the ${ }^{14} \mathrm{C}$ cosmogenic radioisotopes will occur around 2804 A. D. and the next maximum around 3963 A. D..

\section{Acknowledgment}

The authors would like to thank the two referees for very useful suggestions and comments.

\section{References}

Abreu, J. A., Beer, J., Ferriz-Mas, A., McCracken, K. G., Steinhilber, F.: Is there a planetary influence on solar activity? Astron. Astrophys., 548, A88, 2012.

Abreu, J. A., Albert, C., Beer J., Ferriz-Mas A., McCracken K. G., Steinhilbe F.: Response to: "Critical Analysis of a Hypothesis of the Planetary Tidal Influence on Solar Activity". Solar Physics 289, 2343-2344, 2014.

Adolphi, F., Muscheler, R., Svensson, A., Aldahan, A., Possnert, G., Beer, J., Sjolte, J., Björck, S., Matthes, K., Thiéblemont, R.: Persistent link between solar activity and Greenland climate during the Last Glacial Maximum. Nature Geoscience 7, 662-666, 2014.

Bard, E., Raisbeck, G. M., Yiou, F., Jouzel, J.: Solar modulation of cosmogenic nuclide production over the last millennium: comparison between ${ }^{14} \mathrm{C}$ and ${ }^{10} \mathrm{Be}$ records. Earth and Planetary Science Letters, 150(3-4), 453-462, 1997.

Bard, E., Raisbeck, G., Yiou, F., Jouzel, J.: Solar irradiance during the last 1200 years based on cosmogenic nuclides. Tellus 52B, 985-992, 2000.

Bray, J. R.: Glaciation and solar activity since the fifth century B.C and the solar cycle. Nature, 220, 672-674, 1968.

Bond, G., Kromer, B., Beer, J., Muscheler, R., Evans, M.N., Showers, W., Hoffmann, S., Lotti-Bond, R., Hajdas, I., Bonani, G.: Persistent solar influence on North Atlantic climate during the holocene. Science 294, 2130-2136, 2001.

Cameron, R. H., Schüssler, M.: No evidence for planetary influence on solar activity. Astron. \& Astrophys., 557, A83, 2013.

Cauquoin, A., Raisbeck, G. M., Jouzel, J., Bard, E. (ASTER Team): No evidence for planetary influence on solar activity 330000 years ago. Astron. \& Astrophys., 561, A132, 2014.

Callebaut, D. K., de Jager, C., Duhau, S.: The influence of planetary attractions on the solar tachocline. Journal of Atmospheric and Solar-Terrestrial Physics 80, 73-78, 2012. 
Callebaut, D. K., de Jager, C., Duhau, S.: Reply to "The influence of planetary attractions on the solar tachocline" by N. Scafetta, O. Humlum, J.E. Solheim, K. Stordahl. Journal of Atmospheric and Solar-Terrestrial Physics 102, 372, 2013.

Charvátová, I.: Can origin of the 2400-year cycle of solar activity be caused by solar inertial motion? Ann. Geophysicae, 18, 399- 405, 2000.

Charvátová, I.: Long-term predictive assessments of solar and geomagnetic activities made on the basis of the close similarity between the solar inertial motions in the intervals 1840-1905 and 1980-2045. New Astron., 14, 25-30, 2009.

Cionco, R. G., Soon, W.: A phenomenological study of the timing of solar activity minima of the last millennium through a physical modeling of the Sun-Planets. New Astronomy, 34, 164-171, 2015.

Creer, K. M.: Geomagnetic field and radiocarbon activity through Holocene time. In: Secular, Solar and Geomagnetic Variations in the last 10000 years. (Eds) Stephenson, F. R. andWolfendale, A. W., Kluwer, Dordrecht, p. 381-397, 1988.

Czymzik, M., Muscheler, R., Brauer, A.: Solar modulation of flood frequency in central Europe during spring and summer on interannual to multi-centennial timescales. Climate of the Past, 12(3), 799-805, 2016.

Damon, P. E.: Production and decay radiocarbon and its modulation by geomagnetic field-solar activity changes with possible implications for global environment. In: Secular, Solar and Geomagnetic Variations in the Last 10000 years, (Eds) Stephenson, F. R. and Wolfendale, A. W., Kluwer, Dordrecht, p. 267-285, 1988.

Damon, P. E., Linick, T. W.: Geomagnetic-heliomagnetic modulation of atmospheric radiocarbon production. Radiocarbon, 28(2A), 266-278, 1986.

Damon, P. E., Cheng, S., Linick, T. W.: Fine and hyperfine structure in the spectrum of secular variations of atmospheric ${ }^{14} C$. Radiocarbon, 31(3), 704-718, 1990.

Damon, P. E., Sonett, C. P.: Solar and terrestrial components of the atmospheric 14C variation spectrum. In: The Sun in Time, (Eds) Sonett, C. P., Giampapa, M. S., Mathews, M. S., The Univesity of Arizona Press, Tucson, p. 360-388, 1992.

Damon, P. E., Jirikowić, J. L.: Radiocarbon evidence for low frequency solar oscillation. In: Rare Nuclear Processes, (Ed) Povinec, P., Proc. 14th Europhysics Conf. on Nuclear Physics, Word Scientific Publishing Co, Singapore, p. 177-202, 1992.

Dansgaard, W., Johnsen, S. J., Clausen, H. B., Dahl-Jensen, D., Gunderstrup, N., Hammer, C., Oeschger, H.: North Atlantic climate oscillations revealed by deep Greenland ice core. In: Climate Processes and Climate Sensivity, (Eds) Hansen, J. E. and Takahashi, T., AGU, Washington, D. C., p. 288-298, 1984.

Darwin, G.: $\quad$ Tides. (Encyclopædia Britannica, Ninth Edition, 1875-89) 1902. http://www.1902encyclopedia.com/T/TID/tides.html

Davis, J. C., Bohling, G.: The search for patterns in ice-core temperature curves. In: Gerhard, L.C., Harrison, W.E., Hanson, B.M. (Eds.), Geological Perspectives of Global Climate Change, pp. 213-229, 2001.

Dergachev, V., Chistyakov, V.: Cosmogenic radiocarbon and cyclical natural processes. Radiocarbon, 37(2), 417-424, 1995. 
Divari, N. B.: Charged Dust Particles in Interplanetary Space. Soviet Astronomy, 10, 151-154, 1966.

Doodson, A. T.: The Harmonic Development of the Tide-Generating Potential. Proceedings of the Royal Society of London. Series A, 100 (704), 305-329, 1921.

Elsasser, W., Ney, E. P., Winckler, J. R.: Cosmic ray intensity and geomagnetism. Nature, 178, 1226-1227, 1956.

Ermakov, V. I., Okhlopkov, V. P., Stozhkov, Yu. I.: The impact of cosmic dust on the earth's climate. Moscow University Physics Bulletin, 64(2), 214-217, 2009a.

Ermakov, V. I., Okhlopkov, V. P., Stozhkov, I. Yu.: Influence of cosmic rays and cosmic dust on the atmosphere and Earth's climate. Bulletin of Russian Academy of Sciences: Physics, 434-436, 2009b.

Fairbridge, R. W., 1984. Planetary periodicities and terrestrial climate stress. In Mörner and Karlén (1984), 509-520, 1984.

Fairbridge, R. W., Sanders, J. E.: 1987. The Sun's orbit, AD 250-2050: basis for new perspectives on planetary dynamics and Earth-Moon linkage. In Rampino et al. (1987), 446-471, bibliography 475-541, 1987.

Folkner, W. M., Williams, J. G., Boggs, D. H., Park, R. S., Kuchynka, P.: The Planetary and Lunar Ephemerides DE430 and DE431. IPN Progress Report 42-196, 2014. http://ipnpr.jpl.nasa.gov/progress_report/42-196/196C.pdf

Folkner, W. M.: Planetary Ephemeris DE432. Jet Propulsion Laboratory Memorandum IOM 392R-14-003, 2014.

Gervais, F.: Anthropogenic CO2 warming challenged by 60-year cycle. Earth-Science Reviews, 155, 129-135, 2016.

Ghil, M., Allen, M. R., . Dettinger, M. D., Ide, K., Kondrashov, D., Mann, M. E., Robertson, A. W., Saunders, A., Tian, Y., Varadi, F., Yiou, P.: Advanced Spectral Methods for Climatic Time Series. Reviews of Geophysics 40, 1003, 2002. SSA-MTM Toolkit for spectral analysis (http://research.atmos.ucla.edu/tcd//ssa/).

Gregori, G. P.: Galaxy - Sun - Earth relations. The origin of the magnetic field and of the endogenous energy of the Earth, with implications for volcanism, geodynamics and climate control, and related items of concern for stars, planets, satellites, and other planetary objects. A discussion in a prologue and two parts. Beiträge zur Geschichte der Geophysik und Kosmischen Physik, Band 3, Heft 3, 471 pp., 2002.

Grinsted, A., Moore, J. C., Jevrejeva, S.: Application of the cross wavelet transform and wavelet coherence to geophysical time series, Nonlin. Process. Geophys., 11, 561566, 2004.

Goslar, T., Wohlfarth, B., Björck, S., Possnert, G., Björck, J.: Variations of atmospheric ${ }^{14} C$ concentrations over the Allerød- Younger Dryas transition. Climate Dynamics, 15, 29-42, 1999.

Hood, L. L., Jirikowić, J. L.: Recurring variations of probable solar origin in the atmospheric ${ }^{14} C$ time record. Geophys. Res. Letts., 17, 85, 1990.

Hoyt, D. V., Schatten, K. H.: The Role of the Sun in the Climate Change. Oxford Univ. Press, New York, 1997. 
Holm, S.: On the alleged coherence between the global temperature and the Sun's movement. J. Atmos. Sol.-Terr. Phys., 110-111, 23-27, 2014a.

Holm, S.: Prudence in estimating coherence between planetary, solar and climate oscillations. Astrophys. Space Sci., 357:106, 1-8, 2015.

Howell, E.: 2014. Weak Sun poses radiation risk for Mars-bound astronauts. Space.com, issued Nov $08,2014$.

Houtermans, J. C.: Geophysical interpretation of Bristlecone pine radiocarbon measurements using a method of Fourier analysis of unequally spaced data. Ph.D. Thesis, Univ. of Bern, 1971.

Humlum, O., Solheim, J.-E., Stordahl, K.: Identifying natural contributions to late Holocene climate change. Global and Planetary Change, 79(1), 145-156, 2011.

Hung, C.-C.: Apparent relations between solar activity and solar tides caused by the planets. NASA report/TM-2007-214817 (2007). Available at http://ntrs.nasa.gov/search.jsp?R=20070025111

Kerr, R. A.: A variable sun paces millennial climate. Science 294, 1431-1433, 2001.

Kirby, J.: Cosmic Rays and Clouds. Surveys in Geophysics, 28, 333-373, 2007.

Kromer, B., Spurk, M., Remmele, S., Barbetti, M., Toniello, V.: Segments of atmospheric ${ }^{14} C$ change as devided from Late Glacial and Early Holocene floating tree-ring series. Radiocarbon, 40(1), 351-358, 1998.

Jakubcová, I., Pick, M.: The planetary system and solar-terrestrial phenomena. Stud. Geophys. Geod., 30, 224-235, 1986.

Jose, P. D.: Sun's motion and sunspots. Astron. J., 70, 193-200, 1965.

Lal, D.: Theoretically expected variations in the terrestrial cosmicray production rates of isotopes. In: Solar-terrestrial relationships and the Earth environment in the last millennia, (Ed) Castagnoli, G. C., North-Holland Press, Amsterdam, p. 216-233, 1988.

Levina, T. P., Orlova, L. A.: Holocene climatic rhythms of southern West Siberia. Russian Geology and Geophysics, 34, 36-51, 1993.

Loehle, C., Scafetta, N.: Climate Change Attribution Using Empirical Decomposition of Climatic Data. The Open Atmospheric Science Journal 5, 74-86, 2011.

Manzi, V., Gennari R., Lugli S., Roveri M., Scafetta N., Schreiber C.: High-frequency cyclicity in the Mediterranean Messinian evaporites: evidence for solar-lunar climate forcing. Journal of Sedimentary Research 82, 991-1005, 2012.

Marcott, S. A., Shakun, J. D., Clark, P. U., Mix, A. C.: A reconstruction of regional and global temperature for the past 11,300 years. Science, 339(6124), 1198-1201, 2013.

Mayewski, P. A., Rohling, E. E., Stager, J. C., Karlen, W., Maasch, K. A., Meeker, L. D., Meyerson, E. A., Gasse, F., van Kreveld, S., Holmgren, K., Lee-Thorp, J., Rosqvist, G. Rack, F., Staubwasser, M., Schneider, R. R. and Steig, E. J.: Holocene climate variability. Quaternary Research, 62(3), 243-255, 2004.

Mazzarella, A., Scafetta, N.: Evidences for a quasi 60-year North Atlantic Oscillation since 1700 and its meaning for global climate change. Theoretical Applied Climatology 107, 599-609, 2012. 
McCracken, K. G., Dreschhoff, G. A. M, Smart, D. F. , Shea, M. A.: Solar cosmic ray events for the period 1561-1994: 2. The Gleissberg periodicity. J. Geophys. Res 106.21: 599-21, 2001.

McCracken, K. G., Beer, J., Steinhilber, F., Abreu, J.: A phenomenological study of the cosmic ray variations over the past 9400 years, and their implications regarding solar activity and the solar dynamo. Sol. Phys., 286, 609-627, 2013.

McCracken, K. G., Beer, J., Steinhilber, F., Abreu, J.: Evidence for planetary forcing of the cosmic ray intensity and solar activity throughout the past 9400 years. Sol. Phys., 286(2), 609-627, 2014.

Melchior, P.: The Tides of the Planet Earth. Pergamon Press, Oxford, 1978.

Milanković, M.: Mathematische Klimalehre und Astronomische Theorie der Klimaschwankungen, Handbuch der Klimatologie, Band I, Teil A,Berlin, Verlag von Gebrüder Borntraeger, 1930.

Mörner, N.-A.: Global Change and interaction of Earth rotation, ocean circulation and paleoclimate, An. Brazilian Acad. Sc., 68 (Supl. 1), 77-94, 1996.

Mörner, N.-A.: Planetary beat and solar-terrestrial responses. Pattern Recogn. Phys., 1, 107-116, 2013.

Mörner, N.-A.: The Approaching New Grand Solar Minimum and Little Ice Age Climate Conditions. Natural Science, 7, 510-518, 2015.

Mortari, R.: I ritmi segreti dell'universo, 336 pp., (3rd ed.) Aracne editrice s.r.l., Roma. The 1st ed. appeared in 1988, the 2nd ed. in 1999, 2010.

Mungan, C. E.: Another comment on 'Eccentricity as a vector'. Eur. J. Phys., 26, L7-L9, 2005.

O'Brien, K.: Secular variations in the production of cosmogenic isotopes in the Earth's atmosphere. J. Geophys. Res., 84, 423-431, 1979.

O’Brien, S. R., Mayewski, P. A., Meeker, L. D., Meese, D. A., Twickler, M. S., Whitlow, S. I.: Complexity of Holocene climate as reconstructed from a Greenland ice core. Science, 270, 1962-1964, 1995.

Oeschger, H., Siegenthaler, U., Schotterer, U., Gugelmann, A.: A box diffusion model to study the carbon dioxide exchange in nature. Tellus, 27, 168-192, 1975.

Ollila, A.: Cosmic theories and greenhouse gases as explanations of global warming. Journal of Earth Sciences and Geotechnical Engineering 5(4): 27-43, 2015.

Ogurtsov, M.G., Nagovitsyn, Y.A., Kocharov, G.E., Jungner, H.: Long-period cycles of the Sun's activity recorded in direct solar data and proxies. Solar Physics 211, 371-394, 2002.

Owens, M. J., Forsyth, R. J.: The Heliospheric Magnetic Field. Living Rev. Solar Phys., 10, $5,2013$.

Pestiaux P., Duplessy, J. C., van der Mersch, I., Berger, A.: Paleoclimatic variability at frequencies ranging from 1 cycle per 10000 years to 1 cycle per 1000 years: Evidence for nonlinear behaviour of the climate system. Climatic Change, 12(1), 9-37, 1988.

Piovan, L., Milani, F.: Moto del Sole intorno al baricentro del Sistema Solare (Solar motion around the solar system barycentre). Astronomia, 3, 38-44, 2006.

Phillips, T.: Spacecraft sees solar storm engulf Earth, Science@NASA, issued Aug 18, 2011. 
Poluianov, S., Usoskin, I.: Critical Analysis of a Hypothesis of the Planetary Tidal Influence on Solar Activity. Solar Physics 289, 2333-234, 2014.

Potgieter, M. S.: Solar Modulation of Cosmic Rays. Living Rev. Solar Phys., 10, 3-66, 2013.

Puetz, S. J., Prokoph, A., Borchardt, G., Mason, E. W.: Evidence of synchronous, decadal to billion year cycles in geological, genetic, and astronomical events. Chaos Solitons Fractals, 62-63, 55-75, 2014.

Reimer, P. J., Baillie, M. G. L., Bard, E., Bayliss, A., Beck, J. W., Bertrand, C. J. H., Blackwell, P. G., Buck, C. E., Burr, G. S., Cutler, K. B., Damon, P. E., Edwards, R. L., Fairbanks, R. G., Friedrich, M., Guilderson, T. P., Hogg, A. G., Hughen, K. A., Kromer, B., McCormac, G., Manning, S., Bronk Ramsey, C., Reimer, R. W., Remmele, S., Southon, J. R., Stuiver, M., Talamo, S., Taylor, F. W., Plicht, J. V. D., Weyhenmeyer, C. E.: IntCal04 terrestrial radiocarbon age calibration, 0-26 cal kyr BP. Radiocarbon, 46 (3), 1029-1058, 2004. https://www.radiocarbon.org/IntCal04.htm

Salvador, R.: A Mathematical Model of the Sunspot Cycle for the Past 1000 yr. Pattern Recognition in Physics, 1, 117-122, 2013.

Scafetta, N., Grigolini, P., Imholt, T., Roberts, J. A., West, B. J.: Solar turbulence in earth's global and regional temperature anomalies. Physical Review E 69, 026303, 2004.

Scafetta, N., West, B. J.: Phenomenological solar signature in 400 years of reconstructed Northern Hemisphere temperature record. Geophysical Research Letters 33, L17718, 2006.

Scafetta, N.: Empirical analysis of the solar contribution to global mean air surface temperature change. Journal of Atmospheric and Solar-Terrestrial Physics 71, 1916-1923, 2009.

Scafetta, N.: Empirical evidence for a celestial origin of the climate oscillations and its implications. J. Atmos. Sol.-Terr. Phys., 72(13), 951-970, 2010.

Scafetta, N.: Multi-scale harmonic model for solar and climate cyclical variation throughout the Holocene based on Jupiter-Saturn tidal frequencies plus the 11-year solar dynamo cycle. J. Atmos. Sol.- Terr. Phys., 80, 296-311, 2012a.

Scafetta, N.: Does the Sun work as a nuclear fusion amplifier of planetary tidal forcing? A proposal for a physical mechanism based on the mass-luminosity relation. J. Atmos. Sol.-Terr. Phys., 81-82, 27-40, 2012 b.

Scafetta, N.: A shared frequency set between the historical mid-latitude aurora records and the global surface temperature. Journal of Atmospheric and Solar-Terrestrial Physics 74, 145-163, 2012c.

Scafetta, N.: Discussion on climate oscillations: CMIP5 general circulation models versus a semi-empirical harmonic model based on astronomical cycles. Earth-Sci. Rev., 126, 321-357, 2013.

Scafetta, N.: Discussion on the spectral coherence between planetary, solar and climate oscillations: a reply to some critiques. Astrophys. Space Sci., 354, 275-299, 2014a.

Scafetta, N.: The complex planetary synchronization structure of the solar system. Pattern Recognition in Physics 2, 1-19, 2014b. DOI: 10.5194/prp-2-1-2014.

Scafetta, N.: Multi-scale dynamical analysis (MSDA) of sea level records versus PDO, AMO, and NAO indexes. Climate Dynamics, 43, 175-192, 2014c. 
Scafetta, N.: High resolution coherence analysis between planetary and climate oscillations. Advances in Space Research, 2016. DOI: 10.1016/j.asr.2016.02.029

Scafetta, N., Humlum, O., Solheim, J.-E, Stordahl, K.: Comment on "The influence of planetary attractions on the solar tachocline" by Callebaut, de Jager and Duhau. Journal of Atmospheric and Solar-Terrestrial Physics 102, 368-371, 2013.

Scafetta, N., Willson, R. C.: Planetary harmonics in the historical Hungarian aurora record (1523-1960). Planet. Space Sci., 78, 38-44, 2013a.

Scafetta, N., Willson, R. C.: Empirical evidences for a planetary modulation of total solar irradiance and the TSI signature of the 1.09-year Earth-Jupiter conjunction cycle. Astrophys. Space Sci., 348(1), 25-39, 2013b.

Sharp, G. J.: Are Uranus \& Neptune Responsible for Solar Grand Minima and Solar Cycle Modulation? International Journal of Astronomy and Astrophysics, 3, 260-273, 2013.

Shaviv, N. J., Prokoph, A., Veizer, J.: Is the Solar System's Galactic Motion Imprinted in the Phanerozoic Climate? Scientific Reports 4, 6150, 2014. DOI: 10.1038/srep06150

Siegenthaler, U., Heimann, M., and Oeschger, H.: ${ }^{14} C$ variations caused by changes in the global carbon cycle. Radiocarbon, 22, 177-191, 1980.

Smythe, C.M., Eddy, J.A., 1977. Planetary tides during maunder sunspot. Nature 266, 434-435, 1977.

Solanki, S.K., Usoskin, I.G., Kromer, B., Schussler, M., and Beer, J.: Unusual activity of the Sun during recent decades compared to the previous 11,000 years, Nature, 431, 1084-1087, 2004.

Solheim, J.-E.: Signals from the planets, via the Sun to the Earth. Pattern Recogn. Phys., 1, 177-184, 2013.

Sonett, C. P.: Very long solar periods and radiocarbon record. Rev. Geophys. S. Phys., 22(3), 239-254, 1984.

Stefani, F., Giesecke, A.,Weber, N., Weier, T.: Synchronized Helicity Oscillations: A Link Between Planetary Tides and the Solar Cycle? Solar Physics (2016). doi:10.1007/s11207-016-0968-0

Stocker, T. F., Wright, D. G.: Rapid changes in ocean circulation and atmospheric radiocarbon. Paleoceanography, 11, 773-795, 1996.

Steinhilber, F., Beer, J., Fröhlich, C.: Total solar irradiance during the Holocene. Geophysics Research Letters 36, L19704, 2009.

Stuiver, M., Quay, P. D.: Changes in atmospheric carbon-14 attributed to a variable Sun. Science, 207, $11-19,1980$.

Stuiver, M., Reimer, P. J., Bard, E., Beck, J. W., Burr, G. S., Hughen, K. A., Kromer, B., McCormac, G., van der Plicht, J., Spurk, M.: IntCal98 radiocarbon age calibration, 24,000-0 cal BP. Radiocarbon 40(3), 1041-83, 1998 .

Svensmark, H.: Influence of Cosmic Rays on the Earth's climate. Phys. Rev. Lett., 81, 5027-3030, 1998.

Svensmark, H., Bondo, T., Svensmark, J.: Cosmic ray decreases affect atmospheric aerosols and clouds, Geophys. Res. Lett., 36, L15101, 2009. doi:10.1029/2009GL038429

Svensmark, J., Enghoff, M. B., Svensmark, H.: Effects of cosmic ray decreases on cloud microphysics. Atmos. Chem. Phys. Discuss., 12, 3595-3617, 2012. 
Tan, B., Cheng, Z.: The mid-term and long-term solar quasiperiodic cycles and the possible relationship with planetary motions. Astrophys. Space Sci., 343, 511-521, 2013.

Tattersall, R.: The hum: log-normal distribution and planetary-solar resonance. Pattern Recogn. Phys., 1, 185-198, 2013a.

Tattersall, R.: Apparent relations between planetary spin, orbit, and solar differential rotation. Pattern Recognition Phys., 1, 199-202, 2013b.

Tinsley, B. A.: The global atmospheric electric circuit and its effects on cloud microphysics. Reports on Progress in Physics, 71, 066801, 2008.

Usoskin, I. G., Gallet, Y., Lopes, F., Kovaltsov, G. A., Hulot, G.: Solar activity during the Holocene: the Hallstatt cycle and its consequence for grand minima and maxima. Astronomy \& Astrophysics 587, A150, 2016.

Vasiliev, S. S., Dergachev, V. A.: The change of natural radiocarbon level in the Earth's atmosphere over the past 8000 years as a consequence of solar activity, geomagnetic field and climatic factors: 2400-year cycle. Biofizika, 43(4), 681-688, (in Russian), 1998.

Vasiliev, S. S., Dergachev, V. A.: The 2400-year cycle in atmospheric radiocarbon concentration: bispectrum of $14 \mathrm{C}$ data over the last 8000 years. Annales Geophysicae 20, 115-120, 2002.

Vaquero, J.M., Gallego, M.C., García, J. A.: A 250-year cycle in naked-eye observations of sunspots. GRL, 29 (20), 58-1 58-4, 2002.

Wilson, I. R. G.: The Venus-Earth-Jupiter spin-orbit coupling model. Pattern Recogn. Phys., 1, 147-158, 2013.

Wolf, R.: Extract of a letter to Mr. Carrington. Monthly Notices of the Royal Astronomical Society, 19, 85-86, 1859.

Wolff, C. L., Patrone, P.N.: A new way that planets can affect the Sun. Sol. Phys., 266, 227-246, 2010.

Wyatt, M. G., Curry, J. A.: Role for Eurasian Arctic shelf sea ice in a secularly varying hemispheric climate signal during the 20th century. Clim. Dynam., 42, 2763-2782, 2014.

Yu, Z., Chang, S., Kumazawa, M., Furumoto, M., Yamamoto, A.: Presence of periodicity in meteorite falls. National Institute of Polar Research 30, 362-366 Memoirs, Special issue (ISSN 0386-0744), 1983. 